The physics of the colloidal glass transition

This article has been downloaded from IOPscience. Please scroll down to see the full text article.

2012 Rep. Prog. Phys. 75066501

(http://iopscience.iop.org/0034-4885/75/6/066501)

View the table of contents for this issue, or go to the journal homepage for more

Download details:

IP Address: 190.55.69.57

The article was downloaded on 17/05/2012 at 00:32

Please note that terms and conditions apply. 


\title{
The physics of the colloidal glass transition
}

\author{
Gary L Hunter and Eric R Weeks
}

\author{
Department of Physics, Emory University, Math and Science Center 400 Dowman Dr., N201 Atlanta, \\ GA 30322, USA \\ E-mail: weeks@physics.emory.edu
}

Received 26 June 2011, in final form 15 March 2012

Published 16 May 2012

Online at stacks.iop.org/RoPP/75/066501

\begin{abstract}
As one increases the concentration of a colloidal suspension, the system exhibits a dramatic increase in viscosity. Beyond a certain concentration, the system is said to be a colloidal glass; structurally, the system resembles a liquid, yet motions within the suspension are slow enough that it can be considered essentially frozen. For several decades, colloids have served as a valuable model system for understanding the glass transition in molecular systems. The spatial and temporal scales involved allow these systems to be studied by a wide variety of experimental techniques. The focus of this review is the current state of understanding of the colloidal glass transition, with an emphasis on experimental observations. A brief introduction is given to important experimental techniques used to study the glass transition in colloids. We describe features of colloidal systems near and in glassy states, including increases in viscosity and relaxation times, dynamical heterogeneity and ageing, among others. We also compare and contrast the glass transition in colloids to that in molecular liquids. Other glassy systems are briefly discussed, as well as recently developed synthesis techniques that will keep these systems rich with interesting physics for years to come.
\end{abstract}

(Some figures may appear in colour only in the online journal)

This article was invited by P Chaikin.

\section{Contents}

\section{Introduction}

1.1. What is the colloidal glass transition?

1.2. Introduction to the glass transition

1.3. Introduction to colloids

1.4. Basic physics: hard-sphere-like colloids

1.5. More basic physics: diffusion and sedimentation

1.6. Overview of the rest of review

2. Important techniques

2.1. Video microscopy

2.2. Confocal microscopy

2.3. Particle tracking

2.4. Static and dynamic light scattering

2.5. Rheology

2.6. Simulations

3. Features of systems approaching the glass transition

\section{Conclusion}

Acknowledgments

11 References foams
5.1. Soft colloids, sticky particles, emulsions and 


\section{Introduction}

\subsection{What is the colloidal glass transition?}

Imagine you have a bucket of ink. Ink is composed of colourful micrometre-sized particles in water. If you let the water evaporate from the ink, the ink becomes more and more viscous and at some point, it is still damp but no longer flows easily. This increase in viscosity as the water is removed is the colloidal glass transition, and in many respects is analogous to how window glass solidifies as it is cooled from a high temperature.

A colloidal suspension is composed of small solid particles in a liquid, like ink or paint. The key control parameter is the volume fraction $\phi$ : the fraction of volume occupied by the solid particles. Samples with a larger volume fraction will have a larger viscosity, and this viscosity grows quite dramatically as $\phi \rightarrow \phi_{\mathrm{g}} \approx 0.58$. As the glass transition volume fraction $\phi_{\mathrm{g}}$ is approached, the sample's behaviour parallels the glass transition of more traditional (molecular or polymer) glassforming systems [1]. In a chunk of window glass, the atoms are arranged in an amorphous fashion; likewise, in a dollop of glassy colloidal paste, the colloidal particles are arranged in an amorphous way. Given the size of colloidal particles $(\sim 10 \mathrm{~nm}-$ $10 \mu \mathrm{m}$ diameter), they can be studied using a variety of techniques that are difficult or impossible to adapt to molecular glass-formers.

In the following subsections, we introduce basic concepts such as colloids, glasses and some relevant physics, before proceeding with the rest of the review.

\subsection{Introduction to the glass transition}

Upon slow cooling or compression, many liquids freeze - that is, the molecules constituting the liquid rearrange to form an ordered crystalline structure. In general, nucleating a crystal requires undercooling. Some materials can be substantially undercooled without crystal nucleation; alternatively, a sample can be cooled faster than nucleation can occur. In such situations, the liquid is termed supercooled. If the sample is sufficiently cold and cooling is adequately rapid, the material can form a glass: the liquid-like structure is retained but the microscopic dynamics all but cease. This sudden arrest is the glass transition, and the temperature at which it occurs is the glass transition temperature, $T_{\mathrm{g}}$. As the liquid is cooled towards $T_{\mathrm{g}}$, its viscosity rises smoothly and rapidly, and below $T_{\mathrm{g}}$ the sample's viscosity becomes so high that for most practical purposes it is considered a solid. The science of the glass transition is discussed in many review papers [2-10]. Supercooled liquid refers to a system under conditions for which it still flows, but for which the liquid is a metastable state and the thermodynamically preferred state is a crystal. The study of the glass transition then is the study of how a supercooled liquid changes as the temperature $T$ is decreased towards $T_{\mathrm{g}}$, and the study of glasses is the study of materials under conditions where $T<T_{\mathrm{g}}$. Glasses can also be formed at constant $T$ by increasing pressure [11,12].

Calling a glassy material a 'solid' depends on time scales, and perhaps one's patience [13]. Window glass, a vitreous form of silicon dioxide, is of course the quintessential example of glass. It is sometimes claimed that very old windows are thicker at the bottom due to flow of glass. However, the thickness variations in antique windows are the result of a particular manufacturing method rather than the result of the glass flowing over long times [14, 15]. A more instructive example of glassy behaviour and time scales can be seen in pitch, a bituminous tar. Like window glass, pitch is unmistakably solid to the touch-if struck with a hammer, it will shatter. However, for over 80 years a funnel filled with pitch has been dripping at a rate of roughly one drop every 100 months, yielding a very approximate viscosity of $10^{11}$ times that of water. The so-called 'pitch drop experiment' has been housed at the University of Queensland in Brisbane, Australia since 1927 [16].

\subsection{Introduction to colloids}

The term colloid describes a wide range of multiphasic substances composed of particles (solid, liquid or gaseous) roughly $10 \mathrm{~nm}-10 \mu \mathrm{m}$ in size dispersed in a continuous phase. Depending on the state of matter of the various phases, colloids can be divided into several categories, including, but not limited to:

- suspensions/dispersions-solid particles in a liquid (this review's main focus);

- emulsions-liquid droplets in an immiscible liquid;

- foams-gas bubbles in a liquid or solid medium;

- aerosol-liquid droplets or solid particulates in a gas.

Hence, colloid is equally apt to refer to a variety of systems: such as ink, paint, peanut butter, milk and blood (suspensions); Styrofoam ${ }^{\mathrm{TM}}$, shaving cream and ice cream (foams); mayonnaise and hand lotion (emulsions); hair spray and smoke (aerosols). For this range of size, colloids behave as systems of 'classical' particles where quantum mechanical effects can be largely ignored, though it is important to understand the role of quantum phenomena such as van der Waals attractions. More importantly, colloidal particles are small enough that thermal fluctuations are extremely relevant. For example, in a suspension, random collisions between solid particles and solvent molecules lead to Brownian motion, easily observed in experiments.

Aside from the everyday items mentioned previously, industrial processes such as liquid and mineral purification, oil recovery and processing, detergency and even road surfacing employ colloids to varying degrees [17]. Dense colloidal suspensions can be heated and allowed to flow while retaining some rigidity. Hence, they can be moulded, extruded and subsequently solidified to form a multitude of components. The manufacture of many types of optics, insulators, bricks and ceramics involve colloids [18].

While these examples span a wide range of useful materials, colloids also find use in laboratories as models for phases of matter. During the late 1960s and early 1970s, experiments demonstrated that structures in colloidal suspensions can be analogous to those in atomic systems [19-21], leading to extensive use of colloids over the next decade as model liquids and crystals [22-30]. In 1982, Lindsay 


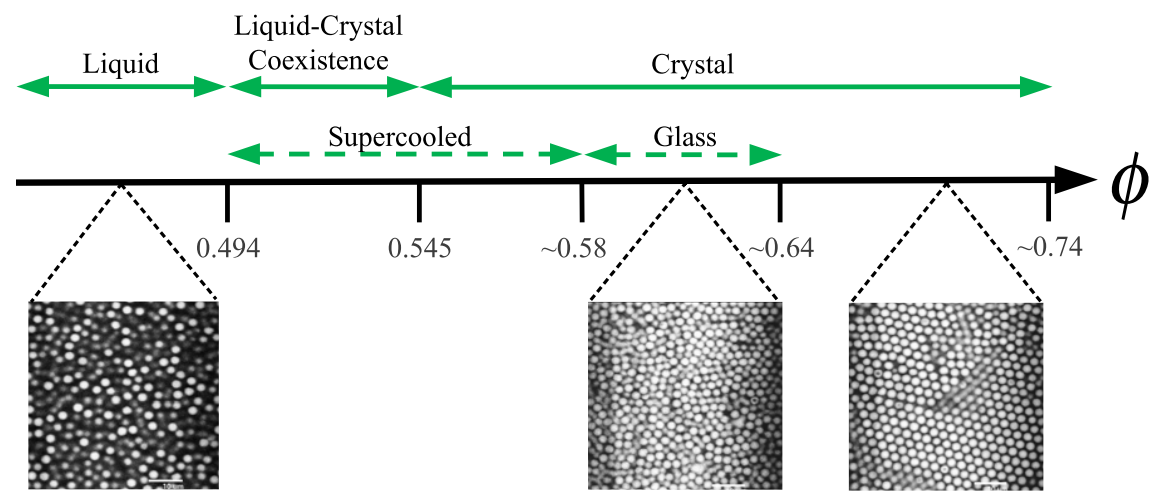

Figure 1. Top: phase diagram of monodisperse hard spheres as a function of volume fraction, $\phi$. Solid arrows indicate equilibrium states, whereas dashed arrows are non-equilibrium states. Note that the existence of the glassy state requires some polydispersity (at least $8 \%$ ); a more monodisperse sample will eventually crystallize [40-44]. However, polydispersity also shifts the boundaries between liquid and crystal to slightly higher values $[40,45,46]$. On the other hand, adding a slight charge to the particles shifts the phase boundaries to lower values [47,48]. More highly charged particles shifts the phase boundaries substantially [47,49]. Bottom: confocal micrographs of the analogous phases in a colloidal suspension with $5 \%$ polydispersity.

and Chaikin combined two different sizes of charged colloidal particles and observed a glassy phase (amorphous structure, finite rigidity) [13] in agreement with subsequent simulations [31]. Later in 1986 and 1987, experiments by Pusey and van Megen demonstrated a hard-sphere colloidal glass transition in a concentrated sample of uncharged colloids [30, 32, 33].

\subsection{Basic physics: hard-sphere-like colloids}

Perhaps the simplest interaction between two particles is that of hard-spheres [34]. If $r$ defines the distance between two sphere centres, and $\sigma$ is the sum of the two sphere radii, the hard-sphere potential is given by

$$
V(r)= \begin{cases}\infty, & \text { if } r \leqslant \sigma, \\ 0, & \text { otherwise, }\end{cases}
$$

which is to say that the only restriction placed upon the system is that particles cannot interpenetrate. Hence, all allowable configurations have identically zero potential energy. From a viewpoint of statistical mechanics, this implies that the free energy, $F=U-T S=(3 / 2) N k_{\mathrm{B}} T-T S=($ const $-S) T$, is governed entirely by entropy $[35,36]$, which for monodisperse systems (systems of a single-particle size) means that the only control parameter is volume fraction [1,37-39]. Volume fraction, $\phi=N V_{\mathrm{p}} / V$, is a dimensionless analogue of particle number density, where $N$ is the number of particles in the system, $V_{\mathrm{p}}$ is the single-particle volume and $V$ is the total system volume. (Note that the particle size controls how fast a system evolves due to diffusion, but does not control the phase behaviour; see section 1.5 for a discussion of particle size effects.)

The phase diagram for hard spheres is shown in figure 1 as a function of $\phi$. Below the freezing point, $\phi_{\text {freeze }}=$ 0.494 , the suspension is a liquid. Forcing the system into a supercooled or glassy state requires increasing $\phi$ fast enough to avoid crystallization. The supercooled region persists between $0.494 \leqslant \phi<\phi_{\mathrm{g}} \approx 0.58$, whereas the glassy region lies between $\phi_{\mathrm{g}}<\phi<\phi_{\text {rcp }} \approx 0.64$. The existence of a glassy phase for hard-spheres requires that the sample be somewhat polydisperse, that is, the spheres must have a distribution of sizes [40-44]. The upper bound of the glassy region is the volume fraction at random close packing, $\phi_{\mathrm{rcp}}$, the maximum density of a completely random sphere packing [50-52]; the precise value of $\phi_{\text {rcp }}$ depends on the polydispersity [53]. Above $\phi_{\mathrm{rcp}}$, samples must have domains of crystalline structure, or, preferably from the thermodynamic point of view, the sample may be entirely crystallized. Density can be further increased up the limit of hexagonal close packing, $\phi_{\text {hcp }}=\pi / 3 \sqrt{2} \approx$ 0.74. Hard spheres are often simulated to study the glass transition [43, 44, 54-59].

In many cases, colloidal particles can be considered to be simple hard-spheres $[30,32,60,61]$. The first experimental demonstration of a colloidal hard-sphere glass transition was by Pusey and van Megen in the mid-1980s, who essentially replicated the hard-sphere phase diagram using colloidal samples [30,32]; see, for example, the pictures in figure 1 . The system used in these studies is particularly important for the following reasons: the interactions between particles are of a simple, well-described nature; the simplicity of the interaction allows for comparison with a wide range of systems and easy simulation with computers; they can be studied by techniques such as microscopy, light scattering and rheologythat is, a single sample can be divided and studied by an array of methods. For these reasons, along with the fact that particles are commercially available or can be synthesized readily [62-65], the same types of colloids are still widely used today.

The particles used by Pusey and van Megen were composed of poly(methyl methacryalate) (PMMA) and were sterically stabilized by the addition of a thin surface layer $(\approx 10 \mathrm{~nm})$ of poly-(12 hydroxystearic acid) (PHSA) to minimize aggregation due to van der Waals forces. It is this steric stabilization layer that allows particles to be considered as hard spheres, at least until they are forced close enough to compress the PHSA [66]. These colloids are stable in organic solvents and can be somewhat tailored for experiments, such as being dyed for use in fluorescence microscopy [64, 65, 67]. 
Solvent choice also allows for a greater degree of control. Miscible solvents can be mixed to closely match the density of the particles, minimizing gravitational effects that can be quite significant in studying colloidal glasses [68-70] (discussed below). Solvents can also be blended to closely match the refractive index of the particles, which both lessens van der Waals attractions and allows for use in microscopy or light scattering.

The size range and time scales that accompany colloidal particles are accessible to a variety of experimental techniques such as optical microscopy or light scattering. For example, a micrometre-sized particle in water will diffuse its diameter in about a second, which is easily observable for modern microscopes.

It is important to note that colloidal systems differ from their atomic counterparts in several ways [71,72]. First, short time motion is diffusive in colloids, rather than ballistic. Second, hydrodynamic effects couple particle motions in complex ways [73]. Simulations suggest that these two differences are unimportant for studying the glass transition [74-80] (see also the discussion in section 2.6). A third difference is that colloidal particles are most typically spherically symmetric, and so the geometry of a molecule is usually not replicated in the colloid (see section 5 for recent exceptions). Again, for many cases of interest, this difference is immaterial when studying long-time dynamics; certainly many glass transition simulations study particles with spherically symmetric potentials. A fourth difference is that colloidal suspensions are always slightly polydisperse. This shifts the phase transitions shown in figure 1 to higher values of $\phi[40,45,46]$, and also in general frustrates crystallization $[41,43,44,81,82]$. While this is a distinction in comparison with simple molecular glass-formers, it is less of a distinction with simulations, which often purposefully add polydispersity to frustrate crystallization $[83,84]$. Indeed, as noted in the caption of figure 1, polydispersity appears necessary for a hard-sphere glass transition; monodisperse samples always eventually crystallize [43, 44].

A final distinction is that colloidal samples are influenced by gravity. As observed in one experiment, a sample that was a colloidal glass on Earth spontaneously crystallized in microgravity [69]. Precisely matching the density of particles and solvent also potentially leads to crystallization and can have a striking influence on the ageing of a glassy colloidal sample $[68,70]$ (see section 4.2 for a discussion of ageing). However, the interpretation of these results is controversial. The crystallization seen may be due to differing polydispersity which strongly influences nucleation [43] and may be a confounding variable in these experiments [41, 42, 44, 82, 85]. It may also be due to heterogeneous nucleation at the walls of the sample chambers [30]. Given the robust similarities between colloidal experiments and gravity-free simulations (described in detail in section 2.6), it seems plausible that gravity is typically not a critical factor, but we note this is debatable.

Although PMMA colloids are a widely used model system, they are by no means the only colloidal system used to study the glass transition. Other non-hard-sphere systems will be discussed throughout this review, particularly in section 5 .

\subsection{More basic physics: diffusion and sedimentation}

Two key concepts for thinking about the colloidal glass transition are diffusion and sedimentation. Diffusion sets the rate of the dynamics, and sedimentation can limit the duration of experiments.

The size of colloidal particles is such that they execute Brownian motion due to frequent, random collisions with solvent molecules. Because collisions are random in magnitude and orientation, the average particle displacement in a particular direction $\langle\Delta x\rangle$ is zero. Instead, motion is quantified by the mean square displacement (MSD),

$$
\left\langle\Delta x^{2}\right\rangle=\left\langle[x(t+\Delta t)-x(t)]^{2}\right\rangle=2 D \Delta t .
$$

The angle brackets \langle\rangle indicate an average over all particles and all initial times $t$ for a particular lag time $\Delta t$, and $D$ is the diffusion coefficient. In three dimensions, (2) becomes

$$
\left\langle\Delta r^{2}\right\rangle=6 D \Delta t .
$$

For a single particle of radius $a$ immersed in a solvent of viscosity $\eta$, the diffusion coefficient $D$ is given by the StokesEinstein-Sutherland equation,

$$
D=\frac{k_{\mathrm{B}} T}{6 \pi \eta a},
$$

where $k_{\mathrm{B}}$ is Boltzmann's constant and $T$ is the system temperature [86,87]. This equation shows that $T, \eta$ and $a$ do not play a direct role in the colloidal glass transition; they only influence $D$, which in turn sets a time scale for particle motion. This time scale is the diffusive (or Brownian) time,

$$
\tau_{\mathrm{D}}=\frac{a^{2}}{6 D}=\frac{\pi \eta a^{3}}{k_{\mathrm{B}} T},
$$

which is the average time needed for a particle to diffuse its own radius (using $\left\langle\Delta r^{2}\right\rangle=a^{2}$ in (3)).

For purely diffusive motion, such as in a dilute suspension, the MSD scales with $\Delta t$. Thus, on a log-log plot of $\left\langle\Delta r^{2}\right\rangle$ versus $\Delta t$, one expects a straight line with a slope of unity. The MSD for a colloidal sample at $\phi=0.52$ is shown in figure 2(a). At the smallest $\Delta t$, the MSD shows diffusive behaviour, indicated by the dashed lines. Note that the diffusion constant obtained from this short time-scale motion, $D_{\mathrm{S}}$, differs from that of (4) for $\phi>0$ due to hydrodynamic interactions between the particles [88-92]. $D_{\mathrm{S}}$ drops to approximately $50 \%$ of the value from (4) by around $\phi \approx 0.3$.

As the lag time increases, a plateau develops in the data of figure 2(a) which is indicative of particles being trapped in cages formed by their neighbours. At these time scales, particles are localized and large cumulative motions are suppressed [2,93-98]. At sufficiently long $\Delta t$, particle rearrangements do occur, and so the MSD again increases, eventually recovering diffusive behaviour. Figure $2(b)$ shows the MSDs measured with light scattering for samples at several volume fractions (see section 2.4 for a discussion of light scattering). As $\phi$ increases from left to right, one observes a lengthening of the plateau and thus increasingly slowed dynamics. The plateau heights in figure 2(a) are at $\left\langle\Delta x^{2}\right\rangle / a^{2} \sim 5 \times 10^{-3}$, whereas in $(b)$ they are at $\left\langle\Delta r^{2}\right\rangle / a^{2} \sim 10^{-1}$. For panel $(a)$ the data could be multiplied 

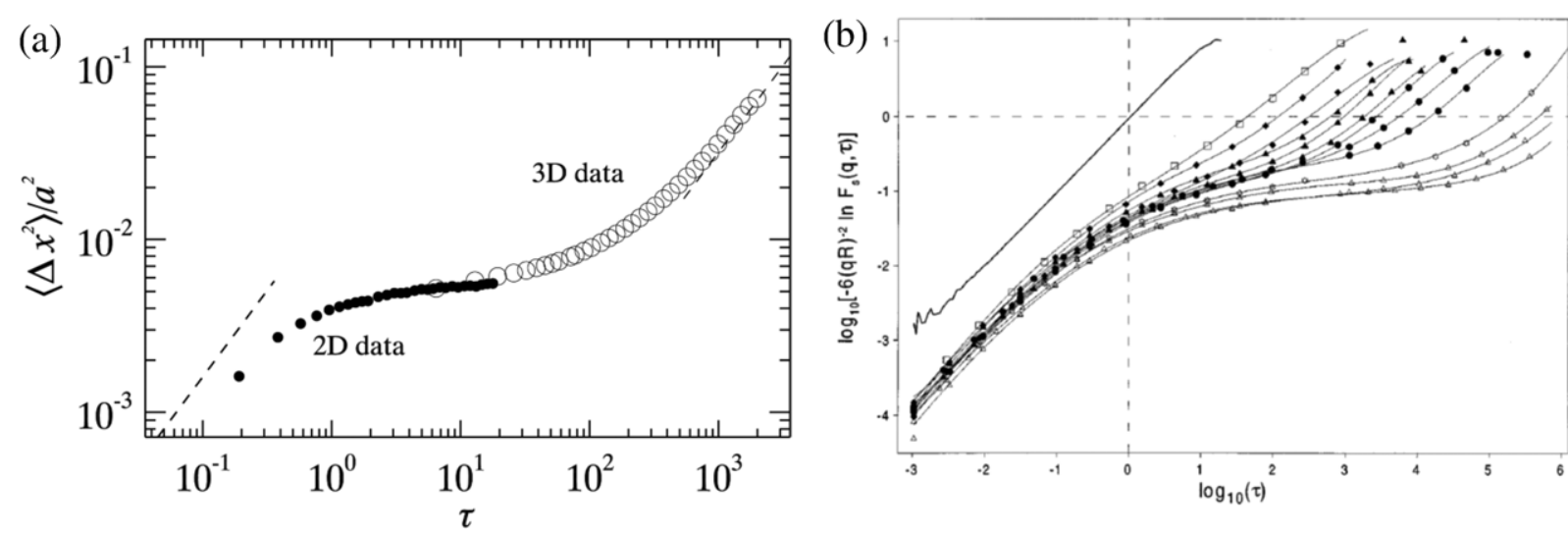

Figure 2. ( $a$ ) An example MSD from a sample with $\phi=0.52$, observed with confocal microscopy. Particles have radius $a=1.18 \mu \mathrm{m}$. Data are from [99]. The horizontal axis has been normalized by the diffusive time $\left(\tau_{\mathrm{D}}=2.79 \mathrm{~s}\right.$ for these particles) and the vertical axis has been normalized by $a^{2}$. The two-dimensional (2D) data (solid circles) are collected at a fixed depth within the 3D sample, while 3D data (open circles) are collected over a fixed sample volume. The 2D data can be acquired more rapidly and probe shorter time scales, which is why the two data sets extend over different ranges in $\Delta t$. The graph shows only the $x$-component of the MSD, but the $y$-and $z$-components are similar. The dashed lines indicate a slope of 1. (b) MSDs measured via light scattering. Time scales are normalized by the diffusive time ( $\tau_{\mathrm{D}}=0.0215 \mathrm{~s}$ ) and measured at $q R=1.3$ using the particle radius $R=200 \mathrm{~nm}$ (here $q$ is used instead of $k$ for the wave vector). The horizontal dashed line corresponds to the particle size $a^{2}$. Volume fraction increases from left to right: $\phi \approx 0$ (solid line), 0.466 (squares), 0.502, 0.519 (closed diamonds), 0.534, 0.538, 0.543, 0.548 (closed triangles), 0.553, 0.558 (closed circles), 0.566 (stars), $0.573,0.578$, 0.583 (open triangles). Figure $(b)$ is reprinted with permission from [100], copyright 1998 by the American Physical Society.

by 3 to convert from $\left\langle x^{2}\right\rangle$ to $\left\langle r^{2}\right\rangle$, giving a plateau height of $\sim 1.5 \times 10^{-2}$, still smaller than the data in panel $(b)$. The difference is very likely due to the different colloidal particles used: those in $(a)$ are slightly charged [99], whereas those in (b) are more nearly ideal hard spheres [100]. The additional repulsion between charged particles would presumably reduce the amplitude of motion within the particle cages, thus reducing the MSD plateau height. The upturns in the MSD for figure $2(a)(\phi=0.52)$ and the solid diamond symbols in figure $2(b)(\phi=0.519)$ are roughly at $\tau \sim 10^{2}$; differences may be due to the differing particle interactions, but are equally likely to be due to differences in $\phi$, given the uncertainty of each experiment's volume fraction measurement [66].

The overall shapes of the MSDs in figure 2 are typical of dense suspensions. In these cases, one often characterizes the system in terms of a long-time diffusion constant, defined as

$$
D_{\mathrm{L}} \equiv \lim _{t \rightarrow \infty} \frac{\left\langle\Delta r^{2}\right\rangle}{6 \Delta t} .
$$

This describes the motions within a system at times after the plateau in the MSD, as shown at large lag times in figure 2.

If the particle size is doubled in a colloidal sample while keeping the volume fraction $\phi$ constant, then the motion slows by a factor of 2 on an absolute scale (from (3) and (4)) and a factor of 8 relative to the particle size (from (5)). However, the overall appearance of the dynamics (liquid-like or glassy) remains the same: more specifically, the behaviour of $\left\langle\Delta r^{2}\right\rangle / a^{2}$ as a function of $\Delta t / \tau_{\mathrm{D}}$ is unchanged. This suggests a useful experimental technique: to effectively explore longtime dynamics, one might use smaller colloidal particles which diffuse faster and reach the long-time behaviour on relatively short experimental time scales. In contrast, video microscopy techniques (section 2.1) work better with slower moving particles, so one typically uses larger particles in such experiments.
The other important consideration for colloidal glass experiments is sedimentation. It is tricky to match the density of the solvent to the density of the colloidal particles, and so over time particles sink to the bottom of a sample chamber (or float to the top). This changes the local volume fraction, the key control parameter, and so sedimentation is important to understand for experiments.

The length scale over which gravity is important is set by balancing the gravitational potential energy $\Delta \rho V_{\mathrm{p}} g z$ with the thermal energy $k_{\mathrm{B}} T$, where $\Delta \rho$ is the density difference between the particle and the solvent, $V_{\mathrm{p}}=\frac{4}{3} \pi a^{3}$ is the volume of the particle, $g$ is the acceleration of gravity and $z$ is a height. Solving this for $z$ gives the scale height

$$
z_{0}=\frac{3}{4 \pi} \frac{k_{\mathrm{B}} T}{\Delta \rho a^{3} g} .
$$

In equilibrium, $\phi$ varies over distances $\sim z_{0}$. In particular, one expects to find $\phi(z) \approx \phi_{0} \exp \left(-z / z_{0}\right)$ for $\phi \ll \phi_{\mathrm{g}}$. If a sample chamber has a height much less than $z_{0}$, then sedimentation can probably be ignored. This is achieved using thin sample chambers, $\sim 200 \mu \mathrm{m}$ thick typically. Alternatively, one can use small particles; as (7) shows, $z_{0} \sim a^{-3}$. More careful matching of the density of the solvent can minimize $\Delta \rho$; here, the chief problem is that solvent and particle densities depend on $T$, so $\Delta \rho$ is only minimized for one particular temperature.

If a colloidal sample is stirred, the initial volume fraction can be fairly homogeneous, and some time is needed to reach the equilibrium volume fraction gradient. This amount of time can be estimated from the sedimentation velocity. The Stokes drag force on a sphere moving with velocity $v$ is

$$
F_{\text {drag }}=6 \pi \eta a v .
$$

The gravitational force acting on a colloidal particle is given by

$$
F_{\text {grav }}=\frac{4}{3} \pi a^{3} \Delta \rho g .
$$


Balancing these two gives the sedimentation velocity as

$$
v_{\text {sed }}=\frac{2}{9} \frac{\Delta \rho g a^{2}}{\eta} .
$$

In practice, the sedimentation velocity is much slower for highvolume fraction samples due to the backflow of the solvent through the sedimenting particles [101-103]. However, $v_{\text {sed }}$ can be used to find a crude estimate for relevant time scales: the volume fraction gradient should be established in time scales of order $z_{0} / v_{\text {sed }} \sim a^{-5} \Delta \rho^{-2}$, for example. Measuring $v_{\text {sed }}$ in a centrifuge (increasing $g$ ) can be used to estimate $\Delta \rho$, again being mindful of the temperature dependence of $\Delta \rho$.

Considerations of diffusion and sedimentation lead to the conclusion that, all else being equal, smaller particles are preferred. However, other experimental considerations often dictate that larger particles be used. Where appropriate, this will be commented on in section 2 which deals with experimental techniques. Another possibility is to use colloids that are much better density matched, and microgel particles are powerful in this regard $[104,105]$. These particles are crosslinked polymers used in a good solvent, where the particle is swollen and permeated with solvent, and thus the density matching is much less an issue.

\subsection{Overview of the rest of review}

The goal of this review is to familiarize the reader with current knowledge of properties of colloidal suspensions in the glassy state $\left(\phi_{\mathrm{g}} \lesssim \phi \lesssim \phi_{\text {rcp }}\right)$ or very near to it $\left(\phi \rightarrow \phi_{\mathrm{g}}\right)$. In particular, we focus mostly on experimental results; for a more theoretical approach, the interested reader should consider other recently published review articles $[1,9,10,72,106]$. The majority of our attention will be given to hard-sphere-like colloids, as many experimental and simulational results concern these systems. We will, however, compare and contrast these observations with other colloidal glasses, as well as with atomic and molecular glasses when appropriate, and describe some theoretical attempts to understand the nature of the glass transition.

Section 2 reviews experimental techniques within the field. It is by no means a complete review of any specific technique, and so references will be given for further reading. Section 3 discusses what is known about the glass transition, that is, $\phi \rightarrow \phi_{\mathrm{g}}$, and section 4 discusses properties of glasses, samples with $\phi>\phi_{\mathrm{g}}$. Section 5 discusses other soft glassy materials, and section 6 is a brief conclusion. Specific discussions of the strengths and weaknesses of colloids as models for glasses are found in section 1.4 (differences between colloids and molecules), section 2.6 (colloids as models compared with simulations as models) and section 6 (focus on strengths of colloids as a model system and discussion of the value of $\phi_{\mathrm{g}}$ ).

\section{Important techniques}

\subsection{Video microscopy}

Microscopy has been used to study colloidal suspensions since the work of Brown and his contemporaries, who reported on the thermal motion of colloidal particles; a good historical account of these observations is [107]. In modern times, the availability, commonality and relative ease-of-use of optical microscopes and video cameras have made video microscopy a popular technique. Whether used in a biology, biochemistry, or physics setting, the mode of operation is the same: a microscope is used to visualize a system; a camera is coupled to the microscope and is used to capture images; and, some type of recording media stores the images for later analysis [108]. Probably the most familiar form of microscopy is brightfield microscopy. Brightfield microscopy relies on scattering or absorption of light by the sample to produce image contrast. Scattering occurs when small differences in the sample's refractive index cause light to deviate from its initial path, leading to a brightened or darkened region in an image. The amount of absorption depends on the material properties of the sample, but image contrast can often be enhanced by the addition of dyes. Modifications of brightfield microscopy include darkfield microscopy, phase contrast microscopy and differential interference contrast microscopy - all of which are effective at improving image contrast when the variations in refractive index are small, such as the case of a living cell (filled mostly with water) in a watery medium [108, 109].

Brightfield microscopy is particularly easy when the sample is quasi-two-dimensional (quasi-2D). For example, quasi-2D colloidal glasses have been studied confined between parallel plates $[110,111]$ or at an interface $[112,113]$. In such experiments, particles always remain in focus and microscopy is quite easy.

A second important type of microscopy is fluorescence microscopy. In this case, the illuminating light is high energy (short wavelength), which excites a dye and causes the emission of longer wavelength light. The advantage of fluorescence microscopy over brightfield is that specific constituents of a sample can be dyed, such as particles in a colloidal suspension, and thus selectively observed [108]. However, the main drawback of using dyes in a sample is that they can lose their ability to fluoresce with increased exposure to light and oxygen - an effect called photobleaching. This means that, over the course of an experiment, the portion of the sample which is being observed will become dimmer. When studying colloidal glasses, the effect of photobleaching is often minor; the time between successive images can be safely set to be on the order of tens of seconds because the dynamics are slow, minimizing the system's exposure to light.

In some cases, the presence of a dye can modify the interactions within a system. For example, in the case of PMMA particles some dyes can leave a small residual electric charge on the particle, causing them to behave as slightly soft spheres rather than hard ones (though this can be countered by adding salts to the solvent [114-116]). Additionally, dyes can decay over time and, over long times, can even diffuse out of the particles and into the solvent, making imaging difficult.

A good general discussion of video microscopy is [117]. Applications of video microscopy to colloidal suspensions are reviewed in $[29,108,118]$.

\subsection{Confocal microscopy}

Conventional optical microscopes are not well suited for 3D microscopy. In order to see deep within a sample, 


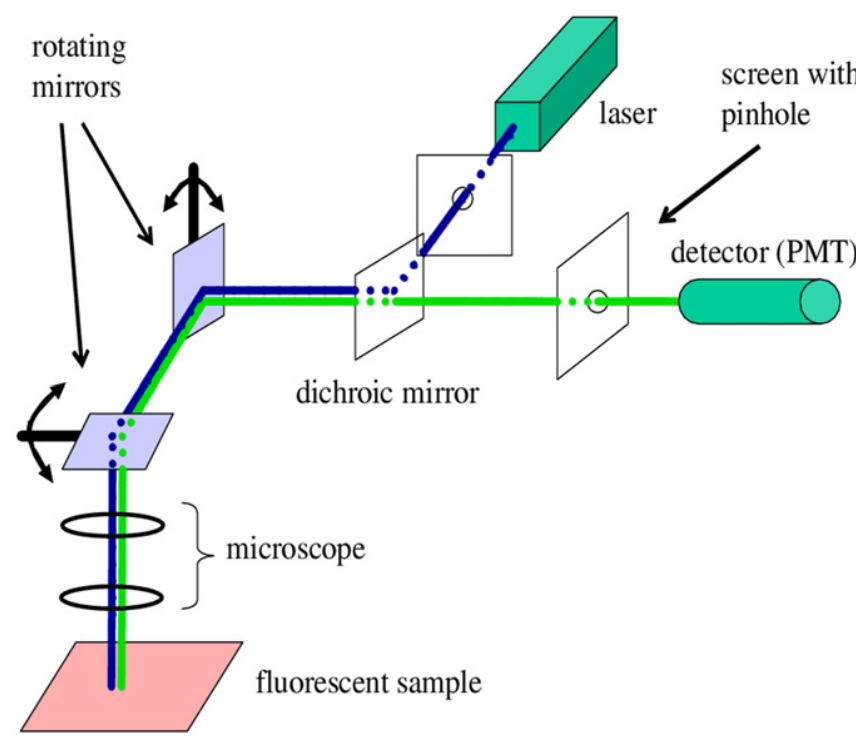

Figure 3. Schematic of a confocal microscope. Rotating mirrors scan the incoming laser light over the region of interest in the sample. The emitted light follows the reverse optical path until arriving at the dichroic mirror, where it passes through the pinhole and into the detector. A dichroic mirror reflects light below a certain wavelength and transmits light above it. Reproduced with permission from [119].

it is necessary to minimize the scattering of light by closely matching the refractive indices of the particles and solvent. Without scattering, conventional optical microscopy is difficult. Fluorescence microscopy overcomes this using the contrast between dyed and undyed portions of the sample to produce an image. This works well for dilute samples, but is poorly suited for dense systems such as colloidal glasses. Because the sample is nearly transparent, objects outside of the focal plane are fluoresced, and stray background light passes readily through the optics and can severely muddle an image: it is hard to distinguish bright particles on a bright background. Confocal microscopes use fluorescence as well, but overcome this limitation with special optics (described below) and are much better suited for studying dense colloidal systems.

The functioning of a confocal microscope hinges on two principles: illumination of a small sample volume $\left(\leqslant 10^{-15} \mathrm{~L}\right)$ and rejection of out-of-focus light [119]. A schematic of a confocal microscope is shown in figure 3. Laser light, shown in black (blue online), passes through a dichroic (dichromatic) mirror and onto rotating mirrors that scan the light in the horizontal planes. The light then passes through the microscope optics and excites the fluorescent sample. The emitted light, shown in dark grey (green online), follows the reverse optical path back to the dichroic mirror, where it is reflected onto a screen with a pinhole. The pinhole is placed in the conjugate focal plane of the sample (hence the term confocal), rejecting the vast majority of out-of-focus light and limiting the depth of field [118]. The remaining in-focus light is finally collected by a detector, such as a photomultiplier tube.

Confocal microscopy allows for direct imaging of a sample in two or three dimensions. In two dimensions, an image is constructed by scanning individual points (point scanning microscopes) or lines of points (line scanning
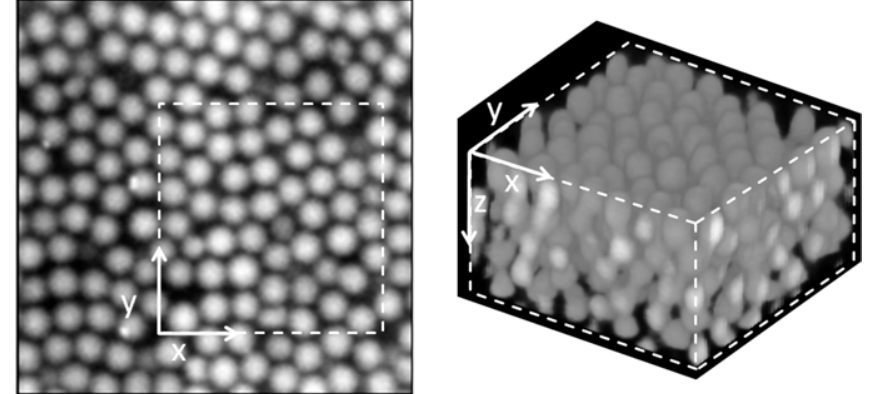

Figure 4. Left: confocal micrograph of a monodisperse colloidal system at volume fraction $\phi \approx 0.63$. The particles have diameter $2 a=2.1 \mu \mathrm{m}$ and the image is taken at the coverslip, where the particles layer against the wall. Right: 3D reconstruction of the boxed region on the left. Here, the image dimensions are $15 \times 15 \times 10 \mu \mathrm{m}^{3}$.

microscopes) over a sample. The highest rates of scanning are achieved with use of an acousto-optical device (AOD), in which one of the mirrors in figure 3 is replaced with a crystal that acts as a diffraction grating whose grating spacing can be tuned with high-frequency mechanical vibrations $[120,121]$. Another option is to use a Nipkow disc, which scans many points simultaneously [122]; these systems achieve high speeds, although more illuminated points slightly increases the background fluorescence detected at any given point.

To obtain 3D images, such as shown in figure 4 , the 2D scanning procedure is quickly repeated while the focal plane is advanced through different depths in the sample. In the fastest modern confocals, 2D images can be collected at rates $\approx 100$ frames s$^{-1}$, and depending on the scanning depth, 3D images can be collected in around $1 \mathrm{~s}$. The specific details (times, pixels) vary from system to system, although it is worth noting that dynamics in dense colloidal systems are quite slow near the glass transition, so even slower confocal microscopes can still obtain adequate images from glassy samples.

The earliest observations of colloids using confocal microscopy were done by Yoshida et al in 1991 [123] and van Blaaderen et al in 1992 [124]. Yoshida et al examined colloidal crystallization near walls, and later studied colloidal gels [125]. van Blaaderen et al demonstrated the utility of fluorescent core-shell particles and confocal microscopy. Core-shell particles are ones with small fluorescent cores and non-fluorescent shells, so that their centres are bright dots that are well separated in the image from other particle centres even at high-volume fractions. The early work of van Blaaderen et al nicely demonstrated the power of confocal microscopy with important proof-of-principle measurements, and hinted at applications using particle tracking [124, 126]. In 1995, van Blaaderen and Wiltzius applied particle identification software to locate the positions of several thousand particles in 3D confocal images to investigate the structure of a colloidal glass [127], sparking much subsequent work [128, 129]. The key 1995 finding was that the structure of a colloidal glass was quite similar to the glassy structure seen in simulations.

More details of applying confocal microscopy to colloidal samples can be found in $[119,130]$, and a good starting point to learn about confocal microscopy is [131]. 


\subsection{Particle tracking}

Particle tracking incorporates various image processing and computational techniques to identify the centroid positions of particles in a given image $[118,132]$. Images can be 2D, as in brightfield or fluorescence microscopy, or 3D, as in confocal microscopy. Repeating the procedures for consecutive images yields a list of coordinates at subsequent times. The coordinates can be used immediately to obtain structural information about a sample, or if dynamic information is desired, the coordinates can be linked together in time to form individual particle trajectories.

In general, the larger a particle is in an image, and the more it contrasts with the background, the more accurate the particle tracking. As mentioned in section 1.5, however, larger particles move slower and are more prone to sedimentation. For many experiments, particle centres can be located with a resolution of approximately $20 \mathrm{~nm}$ in the focal plane, while the out-of-plane resolution is typically no better than $50 \mathrm{~nm}$. Recently, algorithms have been developed that push spatial resolution to $\approx 5 \mathrm{~nm}[133,134]$.

In dilute samples, accurately identifying particles is relatively easy because bright and well-separated particles contrast well with the dark background. In dense samples like colloidal glasses, there are many bright particles in an image and so contrast is usually poorer. Additionally, optical effects such as diffraction can make it difficult to distinguish individual particles when they are very close together. These effects are important to understand and correct, especially when particle motions are very small $[135,136]$. To illustrate, in a sample of $2.4 \mu \mathrm{m}$ diameter PMMA spheres at $\phi=0.52$, Weeks and Weitz observed the majority of particles to move less than $0.2 \mu \mathrm{m}$ over $600 \mathrm{~s}$ [137]. The influence of diffraction can be weakened by increasing the optical resolution using fancier lenses [117], using confocal microscopy (see section 2.2), or with computational techniques $[134,135]$. Hence, with some care as far as optics are concerned, and some fine tuning of particle-tracking parameters, it is often straightforward to study dilute and dense systems with the same techniques.

Combined with video microscopy, particle tracking offers a powerful method to probe the local properties of a sample, which is especially important for understanding structurally or dynamically heterogeneous systems like colloidal glasses. With this technique, one can discuss behaviours of individual particles up to a collection of several thousand. This degree of resolution is not available with light scattering (see section 2.4) or conventional rheology (see section 2.5) where quantities are averaged over thousands to millions of particles. However, such a small statistical sampling can make it difficult to draw conclusions about a system's bulk properties without collecting an overwhelming amount of data.

The main starting point for particle tracking is the original paper by Crocker and Grier [132], and the software described in the paper is available for download on the web [138]. Samples that are flowing or being sheared can also be tracked using pre-treatment of the data; see [139] for details. For a comprehensive assessment of particle tracking, see [134].

\subsection{Static and dynamic light scattering}

Light scattering is a powerful technique for probing the average structure and dynamics of a sample. A laser is aimed at a sample, and the light scattered from the sample at a given angle is detected.

Photons scattered from different portions of the sample interfere with each other, and how this interference (constructive or destructive) depends on angle provides information about the structure of the sample. In particular, this information leads to the static structure factor $S(k)$, the Fourier transform of the particle positions. This is static light scattering (SLS). The scattering wave vector $k$ is given by $k=[4 \pi n / \lambda] \sin (\theta / 2)$, where $\lambda$ is the laser wavelength, $n$ is the index of refraction of the sample medium and $\theta$ is the angle between the incident light and detected light [119, 140,141].

In dynamic light scattering (DLS), the light intensity, $I(t)$, at a fixed angle is monitored as a function of time. The light intensity fluctuates as portions of the sample rearrange, changing the interference pattern of scattered light. In particular, one monitors how the intensity autocorrelation function,

$$
g_{2}(\Delta t)=\frac{\langle I(t+\Delta t) I(t)\rangle_{t}}{\langle I(t)\rangle^{2}},
$$

changes as a function of lag time $\Delta t$. At $\Delta t=0, g_{2}(\Delta t)$ is at a maximum, and decays from this value as the sample evolves. Scattering functions, such as the self-intermediate scattering function shown in figure 5, are related to $g_{2}(\Delta t)$ and are used to quantify dynamics. By measuring the rate of decay, one measures how particles move and can extract information similar to the diffusion coefficient. Probing the dynamics at different $k$ values allows one to determine information about either local or collective particle motion within the sample; most typically, $k$ is chosen to coincide with the peak of the structure factor $S(k)$, which yields information about collective motions. Alternatively, tracer systems can be prepared and single-particle motion probed [100, 142], or else the behaviour at $k \rightarrow \infty$ can be examined which also relates to self-diffusion [89]. For example, the MSDs in figure 2(b) are calculated from the DLS data in figure 5. The autocorrelation function is often calculated over time scales down to $10^{-6} \mathrm{~s}$, allowing a large range of time scales to be measured, as shown in figure 5. For more details about both SLS and DLS, see [140,141].

The main strength of light scattering is that light is scattered from a significant volume within the sample, typically containing millions of particles. The measurement is a very good average of information from all of the particles, whether it be structural information (SLS) or dynamic information (DLS). For DLS, given that the measurement is sensitive to motions corresponding to fractions of $\lambda$, accurate MSDs are straightforward to obtain. However, because of the ensembleaveraging properties, local information is harder to obtain. For example, while calculating the MSD is easy, knowing how individual particle motions are correlated in space is more difficult. A secondary strength of light scattering is that typically particles smaller than those in microscopy experiments can be used, for example $300 \mathrm{~nm}$ radius [54] in one early experiment. These smaller particles are much 


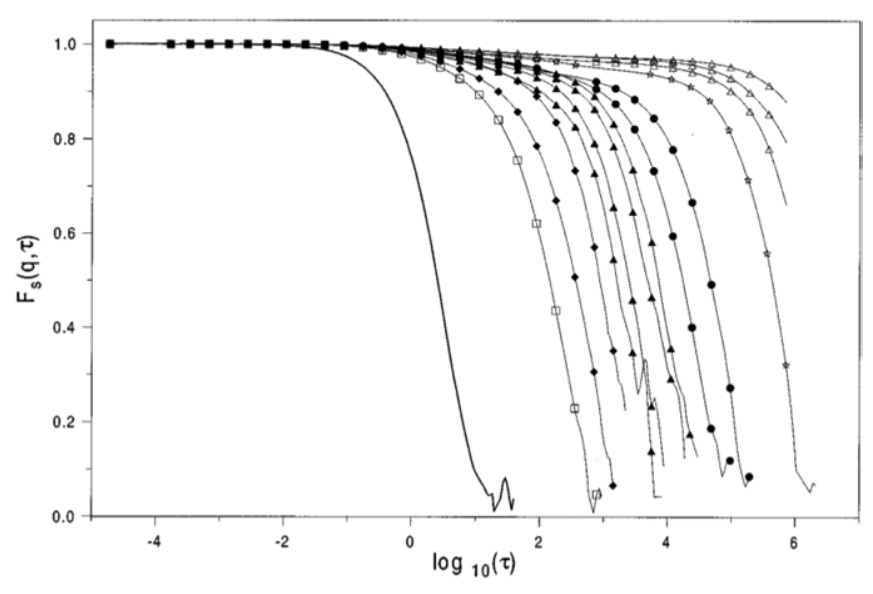

Figure 5. Self-intermediate scattering functions, $F_{\mathrm{s}}(k, \tau)$, as a function of the dimensionless time $\tau=\Delta t / \tau_{\mathrm{D}}$ with $\tau_{\mathrm{D}}=0.0215 \mathrm{~s}$, and measured with wave vector $k R=1.3$. Data and symbols are the same as in figure $2(b)$. Note that an increase in $\phi$ corresponds to an increase in decay time. Reprinted with permission from [100], copyright 1998 by the American Physical Society.

less affected by sedimentation (see section 1.5). Additionally, smaller particles diffuse faster, allowing their motion to be probed over a larger range of time scales.

In 2001, Williams and van Megen devised a clever method to examine binary samples (mixtures of two particle sizes) with SLS and DLS [143]. Their size ratio was $a_{\text {small }} / a_{\text {large }}=$ 0.6. They slightly modified the synthesis method for the two particle types so that they had distinct indices of refraction. By tuning the temperature of the solvent, they could closely match the index of one or the other particle type, and obtain information about each particle species independently. These experiments nicely demonstrated that at a given volume fraction, mixtures of two sizes are more liquid-like than a monodisperse sample [143], in agreement with viscosity measurements $[144,145]$. This is because binary samples can be packed to higher volume fractions than monodisperse samples, so at a given volume fraction, a binary sample has more free volume than a monodisperse sample. A subsequent experiment suggested that the small particles can 'lubricate' the motion of the large particles ( size ratio $a_{\text {small }} / a_{\text {large }}=0.48$ ) [146]. However, theory and simulations indicate that the opposite is true for size ratios $a_{\text {small }} / a_{\text {large }}>0.8$ [58, 147, 148]: for such size ratios, increasing the fraction of small particles can increase the glassiness of the sample. For small size ratios $a_{\text {small }} / a_{\text {large }} \leqslant 0.1$, the depletion force becomes relevant, where the small particles effectively push together the large particles. This can lead to both more and less glassiness, depending on the conditions [149], and is further discussed in section 5.1. Binary samples in general are of interest for understanding multicomponent molecular glasses, and the technique of Williams and van Megen demonstrates how light scattering can be used to study such multi-component systems [143].

In very dense colloidal suspensions, such as those near a glass transition, additional experimental issues arise. One problem is that in a glassy sample, particles do not rearrange significantly, so it is difficult to obtain a proper time-average from the sample. Several techniques have been developed to deal with this situation and are reviewed in [150-152].
A second problem is that light is often scattered from more than one particle before being detected. Again, several techniques have been developed for cases when the light scattered a few times before detection, some of which are reviewed in $[152,153]$. One common technique is diffusive wave spectroscopy (DWS) [154-158] which works when light scatters many times before detection. Here, the light is assumed to be scattered so many times that each photon can be thought to diffuse randomly through the sample before exiting and being detected. Diffusion is straightforward to describe mathematically so, for a given experimental geometry, it is possible to calculate the average number of times a photon has been scattered (and therefore the number of particles from which it scattered). Again, the intensity of light is monitored and its autocorrelation calculated, but now, each particle needs only move a very small fraction of a wavelength before the sum of these motions results in significant decorrelation of the intensity. DWS is thus useful for multiply scattering samples with small motions. Colloidal glasses were studied soon after the development of DWS [157], an early result being that the MSD of densely packed particles is nonlinear in time (as shown in figure 2, for example).

DWS is reviewed in [159]. A useful review paper which briefly discusses differences between DWS and DLS is [160]. Ultra-small-angle neutron and X-ray scattering as applied to colloidal glasses is reviewed in [161]; these techniques can probe structure on length scales of $\sim 1-10 \mu \mathrm{m}$. A recently published book on glasses and dynamical heterogeneity (see section 3.3) contains a chapter by Cipelletti and Weeks which focuses on colloidal glasses [162]. This chapter discusses many details of light scattering. A review paper by Sciortino and Tartaglia compares experimental data with theoretical predictions, with a focus on light scattering data [1].

\subsection{Rheology}

Rheology is the study of how materials flow and deform. A rheological measurement quantifies how solid- or fluid-like a substance is in response to a specific stress [18]; that is, the goal of rheology is to measure elastic and viscous properties of a system. To make such a measurement, one needs a rheometer, a device capable of either creating a constant or oscillatory stress and measuring the resulting rate of deformation, or measuring the stress required to deform a material at a constant rate of strain.

An elementary rheometer is illustrated in figure 6. The device consists of two horizontal plates separated by a distance $h$, where the top plate is mobile and the bottom plate is fixed. On the left of figure 6, an ideal Hookean (elastic) solid is placed inside and the top plate is displaced by a distance $\Delta x$. The stress $\sigma$ (三 force/area) needed to do this is given by the relation

$$
\sigma=G \frac{\Delta x}{h}=G \gamma
$$

This equation defines the shear modulus $G$, where $\gamma=\Delta x / h$ is the strain.

On the right side of figure 6 , the rheometer is filled with a simple fluid, such as water, and the top plate is displaced at a 

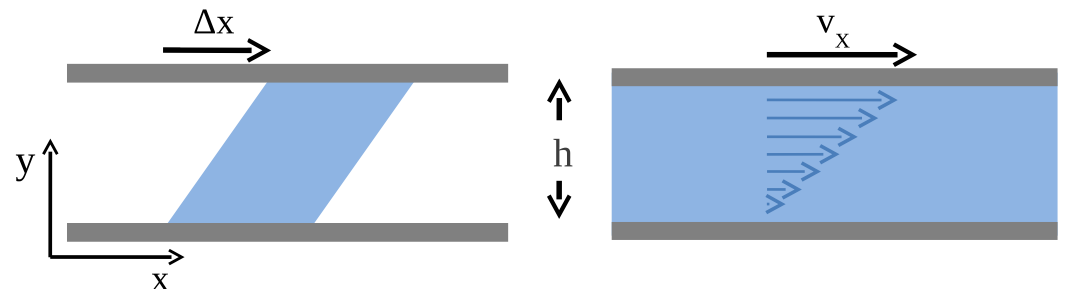

Figure 6. Schematic of a simple rheometer. Left: the top of an ideal solid is displaced $\Delta x$, leading to a strain of $\gamma=\Delta x / h$. Right: a Newtonian fluid is sheared at a rate of $\dot{\gamma}=v_{x} / h$.

constant velocity $v_{x}$. As the top plate moves, it drags the fluid underneath in accordance with the no-slip boundary condition of fluid mechanics. For the same reason, the fluid immediately above the fixed bottom plate is motionless. This creates a steadily decreasing velocity profile (indicated by the arrows). The shear stress needed to maintain the constant velocity of the top plate is given by

$$
\sigma=\eta \frac{\partial v_{x}}{\partial y}=\eta \frac{v_{x}}{h}=\eta \dot{\gamma}
$$

The above equation defines the shear viscosity of a fluid, $\eta=\sigma / \dot{\gamma}$, where $\dot{\gamma}$ is the shear rate. Fluids that adhere to this relation are called Newtonian fluids.

More generally, many materials are termed viscoelastic: they have both a viscous and elastic nature [73, 163-167]. Viscoelasticity can be studied by applying a low amplitude sinusoidally varying strain of the form $\gamma=\gamma_{0} \sin (\omega t)$. As noted above, the elastic stress is proportional to this strain and thus depends on $\sin (\omega t)$, while the viscous stress is proportional to the strain rate and thus depends on $\cos (\omega t)$. For a viscoelastic material one would measure

$$
\sigma(t)=\gamma_{0}\left[G^{\prime}(\omega) \sin (\omega t)+G^{\prime \prime}(\omega) \cos (\omega t)\right],
$$

where the two moduli are the storage modulus $G^{\prime}(\omega)$, and the loss modulus $G^{\prime \prime}(\omega)$. These two moduli in general depend on the frequency $\omega . G^{\prime}$ describes the ability of the material to store elastic energy, while $G^{\prime \prime}$ characterizes energy dissipation. Analogous to a dampened spring, the elastic portion oscillates in phase with the stress, whereas the viscous portion is out of phase by a factor of $\pi / 2$.

It is worth noting that colloidal suspensions are viscoelastic, and so their rheological properties depend on the measurement frequency, $\omega$, as shown in figure 7 [163]. Viscoelastic behaviour has been explored both experimentally and theoretically $[163,168]$. In figure 7 , it can be seen that both $G^{\prime}$ and $G^{\prime \prime}$ rise rapidly near the glass transition over a large range of of $\omega$.

For colloids at the glass transition, the elastic modulus $G^{\prime}(\omega)$ is larger than the loss modulus $G^{\prime \prime}(\omega)$ for a wide range of frequencies, and in particular as $\omega \rightarrow 0$. This latter condition corresponds to solid-like behaviour for a quiescent sample. Related to this is the idea of a yield stress, that a solid-like sample requires a finite stress be applied in order for the sample to flow (flow being defined as $\dot{\gamma}>0$ for a given applied stress) [166].

There exist techniques to measure viscosity and elasticity from video microscopy and particle tracking, and

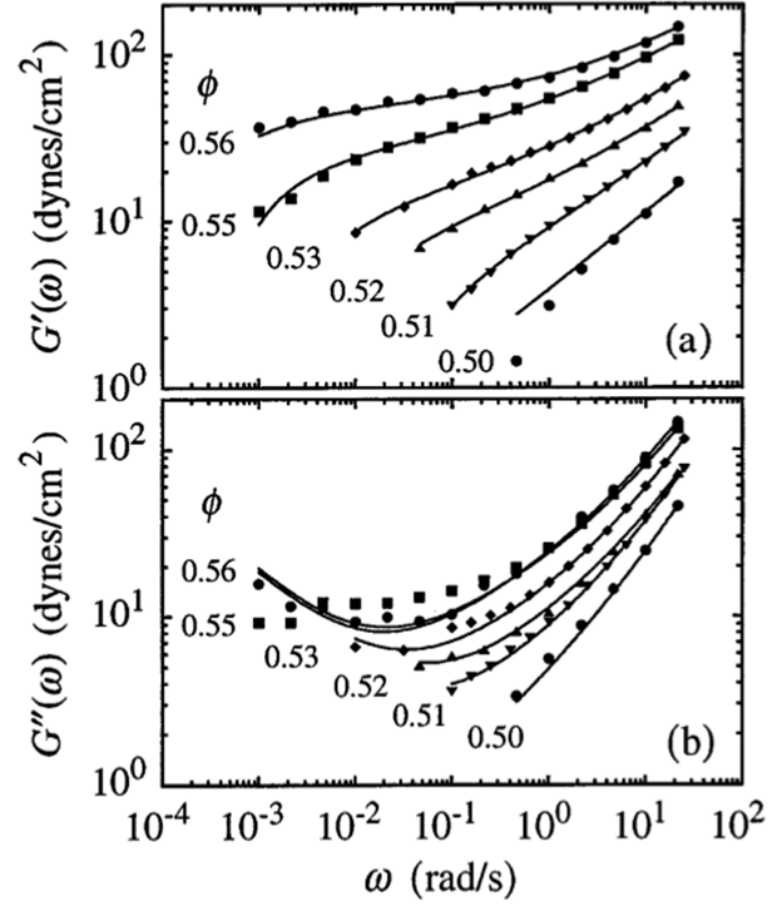

Figure 7. (a) Storage modulus and $(b)$ loss modulus as a function of frequency for colloidal suspensions at different volume fractions. The solid lines are fits to a model based on mode-coupling theory (MCT). Reprinted with permission from [163], copyright 1995 by the American Physical Society.

light scattering; these techniques are collectively termed microrheology [169-173]. It is important to note that microrheology measurements represent local, microscopic properties, whereas rheological measurements involve macroscopic, bulk samples. Applying microrheology methods to dense suspensions requires care in the interpretation [174-184]. Of historical note, the first experiment to use microrheology was by Mason and Weitz in 1995, and this experiment used DWS (section 2.4) to probe colloidal glasses as a test case.

Good reviews about the rheology of colloidal suspensions include [185-189]. More general information about rheology can be found in [18,190-192]. See also the discussion of sheared samples in section 4.3 of this review.

\subsection{Simulations}

As discussed above, colloidal glasses are often considered as model hard-sphere glasses, and complement simulations of 
hard spheres. Likewise, simulations of hard spheres give quite useful insight to colloidal glasses, and in many cases have guided experiments.

It is difficult to simulate a large number of colloidal particles at high-volume fractions taking into account hydrodynamic interactions and interaction potentials; often approximations are desirable or necessary [193]. For that matter, colloidal glasses are themselves only approximate models of molecular glassy materials, so to the extent that colloidal glasses may provide insight into the general glass transition, one hopes that the details are not crucial and that approximations are acceptable. Fortunately, this seems to be the case. First, the microscopic dynamics seem unimportant. Simulations with Brownian dynamics (appropriate for colloids) or Newtonian dynamics (appropriate for simple hard-sphere systems without a solvent) result in similar long-time-scale dynamics [74-80]. Second, the interaction potential seems unimportant. Observations such as dynamical heterogeneities are similar in Lennard-Jones simulations [194-197], hard-sphere simulations [56, 198] and soft sphere simulations [199-202]; see section 3.3. The accumulation of evidence suggests that the specific details of colloidal interactions are not crucial for understanding glassy behaviour. The limitations of the colloidal samples as models (Brownian dynamics with hydrodynamic interactions) may also not be crucial problems for comparing colloidal glasses with molecular glasses. Third, even the dimensionality may be fairly unimportant. Simulations see similar slowing of dynamics in two dimensions and three dimensions, as well as similar particle motions [198, 203,204]. Likewise, colloidal experiments see similar slowing and similar qualitative features in two dimensions $[110,112]$ and three dimensions $[128,129,205]$. One caveat is that preventing ordering is more important in lower dimensions and so binary or polydisperse samples must be used to study glass transitions in two dimensions. However, this also suggests the possibility of better understanding the role of crystallization and frustration by considering higher dimensions; see [206-208] which discuss intriguing results from 4D simulations.

Another consideration for comparing simulations and experiments are finite size effects. In a simulation, often periodic boundary conditions are used. The key assumption, then, is that the box size should be at least twice as big as any structural length scales or dynamical length scales present [8]. Of course, it is possible some of these length scales may be longer than expected-for example, one simulation found evidence for a structural length scale that was three times as large as the more obvious dynamical length scale [209]. Two simple options exist. First, one can conduct simulations for a range of box sizes, and verify that the physics one observes is independent of box size or perhaps scales in some clear way. Second, one can exploit the size dependence to learn something about the sample [210]; see the discussion in section 3.4.

In experiments, finite size effects also can cause problems. In a typical microscopy experiment, 2D images can contain a few hundred particles, or 3D confocal microscopy images can contain a few thousand particles. While the sample chamber may well be much bigger, this still limits the size of dynamical length scales that can be studied; see, for example, the discussion of finite size effects in [129]. Also, samples are often imaged through a glass coverslip, and care must be taken to take the data away from the boundaries; the presence of boundaries introduces layering [57,211-214] and likely changes the dynamics as well [57, 215]. However, microscopy imaging is difficult deep within a sample; here light scattering has an advantage.

Given the similarities between a variety of simulations and the colloidal glass transition, this review paper will not completely survey the literature of simulational studies of the colloidal glass transition because in reality, simulations can be quite relevant for the colloidal glass transition without specifically being simulations of colloids, and this review paper cannot effectively survey all of the simulations of the glass transition. Instead, in subsequent sections of this review, as we describe features of the colloidal glass transition we will discuss the relevant simulation results.

However, some intriguing advantages of simulations are certainly worth noting here. Widmer-Cooper et al demonstrated advantages of the 'iso-configurational ensemble,' where they repeated simulation runs with identical starting positions for particles, but with randomized velocities; this is certainly something well suited to simulation [203, 216]. Their results are described more comprehensively in section 3.3, but briefly, their technique demonstrated that certain regions have a higher propensity for particle motion. Another interesting simulation by Santen and Krauth used non-physical Monte Carlo moves to probe 'equilibrium'-like sample properties for glassy samples [217]. They found that thermodynamic properties were continuous across the transition, evidence that the glass transition is not a thermodynamic transition. The 4D simulations mentioned above led to interesting results as crystallization is much harder in four dimensions, and so even a monodisperse system can have glassy behaviour [206-208]. These three examples_-all using hard particles_-give a sense of the variety of ways that simulations can give unique insight into the colloidal glass transition.

Several textbooks exist which discuss simulation techniques; a good starting point is [218]. Reviews of simulations of the glass transition include [8,219].

A textbook introducing a large variety of methods for studying soft materials is [108]. Many of the techniques discussed above are described in more detail, including microscopy, simulation methods and rheology.

\section{Features of systems approaching the glass transition}

\subsection{Growth of viscosity and relaxation times}

A liquid's viscosity increases upon cooling. If cooling continues into the supercooled regime, the viscosity continues to grow, and at the glass transition is about $10^{13}$ poise [3]. (For comparison, the viscosity of water at room temperature is $0.01 \mathrm{P}$, glycerol is $15 \mathrm{P}$ and honey is $100 \mathrm{P}$ [220].) Analogously, increasing the volume fraction in a colloidal suspension, shown in figure 8 , causes an increase in viscosity. As can be seen, the 


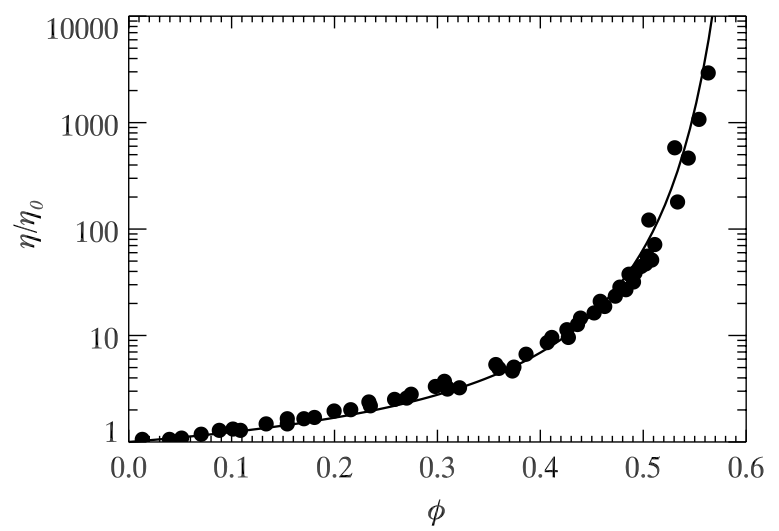

Figure 8. Scaled low shear viscosities at different $\phi$ for various colloidal suspensions of nearly monodisperse hard spheres. The low shear viscosities $(\eta)$ are normalized by the viscosity of the pure solvent $\left(\eta_{0}\right)$. The fit line is to equation (15) with $C=1$, $\phi_{\mathrm{m}}=0.638, D=1.15$. Data taken from [221, 225-227].

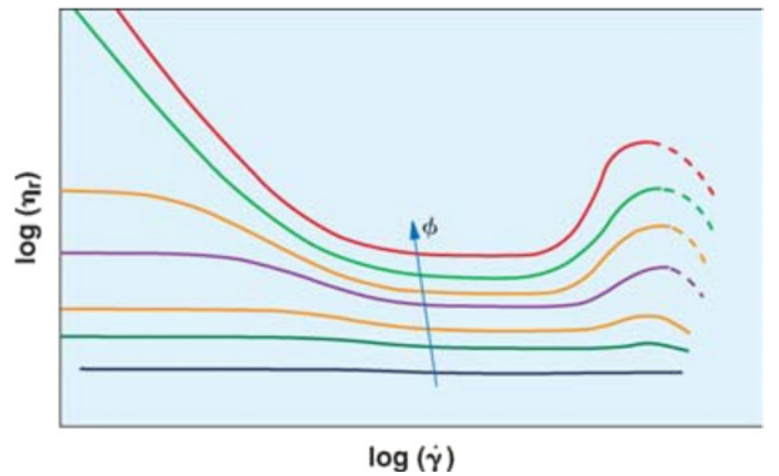

Figure 9. Schematic of shear thinning and shear thickening for colloidal suspensions at various volume fractions. Reprinted with permission from [187].

maximal change in viscosity is only a factor of $10^{4}$; indeed, one critique of the colloidal glass transition as a model for molecular glass transitions is that the viscosity increase is not nearly as great. This discrepancy likely arises for several reasons. First, it is experimentally difficult to load high-volume fraction samples into a rheometer [66,221]. Such a limitation can potentially be overcome using thermosensitive particles, which could be loaded into the rheometer at a temperature where the sample is liquid-like and then thermally changed to a higher volume fraction in situ [222,223]. Second, ensuring that the sample has a well-known and controllable volume fraction can be extremely challenging [66]. Finally, colloidal samples that are sheared too rapidly can shear thin (the apparent viscosity decreases with increasing shear rate) or, at still higher shear rates, shear thicken (an increasing apparent viscosity with increasing shear rate). These trends are indicated qualitatively in figure 9. Shear thinning is more severe for $\phi>0.5$ [28, 224], meaning that experiments at high $\phi$ must be done at extremely low shear rates $(\omega \rightarrow 0$ as described in section 2.5) and low applied stresses to see the correct linear response [178]. Measurements for $\phi \approx 0.6$ would take weeks or years to be done properly [221].

Important early work on the viscosity of colloidal suspensions was performed by Marshall and Zukoski using rheometry [28]. Their system consisted of small silica hard spheres (radius $<300 \mathrm{~nm}$ ) in a solvent of decahydronapthalene. A constant stress rheometer was used to measure viscosity at various applied stresses, enabling an extrapolation of the viscosity to a state of zero stress. For all particle sizes used, they observed an increase in viscosity with $\phi$, with a sudden, apparently divergent increase at volume fractions associated with the glassy phase of hard spheres. They also found that the form of the increase was well described by the Doolittle equation,

$$
\frac{\eta}{\eta_{0}}=C \exp \left[\frac{D \phi}{\phi_{\mathrm{m}}-\phi}\right],
$$

with $C=1.20, D=1.65$ and $\phi_{\mathrm{m}}=0.638$. This equation was first used to describe the temperature dependence of viscosity in molecular liquids approaching the glass transition [228]. The original Doolittle equation was expressed as a function of free volume (which was implicitly a function of temperature). We note that (15) has been modified in a reasonable fashion (see [28] for details) to depend on $\phi$ as shown above, with $\phi_{\mathrm{m}}$ being the maximum packing. At $\phi=\phi_{\mathrm{m}}$ in the above equation, the viscosity diverges. It is intriguing that their observed $\phi_{\mathrm{m}}=0.638$ is close to $\phi_{\mathrm{rcp}}$, where all motion is suppressed. Indeed, the data in figure 8 are also well fit by (15) with a similar $\phi_{\mathrm{m}}$ [221]. This raises questions about whether the glass transition occurs at $\phi_{\mathrm{g}} \approx 0.58$ or possibly at $\phi_{\text {rcp }} \approx 0.64$. We will discuss these questions more comprehensively in section 6, but for now, we note that the viscosity is large at $\phi_{\mathrm{g}}$ and that other quantities, to be discussed next, diverge at $\phi_{\mathrm{g}}$.

The Doolittle model has been critiqued in the past as being oversimplified or perhaps founded on shaky physical arguments [229, 230], and it is possible that other functional forms would fit the data just as well $[165,221]$. The question of which functional form is most appropriate is generic to studying the glass transition. It was noted by Hecksher et al in 2008 that multiple functional forms fit glass transition data (relaxation times as a function of $T$ ) [231,232]. Of these expressions, some have a divergence at finite $T$ while others have no divergence at all. In all cases, the experimental data range over many decades in $\eta$, but are clearly many more decades away from $\eta=\infty$, and so extrapolation is always tricky [233-235].

While the glass transition is associated with a dramatically increased viscosity, it is equally associated with a dramatically increased microscopic relaxation time and decreased diffusivity. For colloids, the long-time self-diffusion coefficient $D_{\mathrm{L}}(\phi)$ approaches zero as $\phi \rightarrow \phi_{\mathrm{g}}$ (see section 1.5 for a discussion of $D_{\mathrm{L}}$ ). A related quantity is the intermediate scattering function $F\left(k_{\mathrm{m}}, \tau\right)$, where the wave vector $k_{\mathrm{m}}$ is often chosen to correspond to the peak of the static structure factor. The decay time for $F\left(k_{\mathrm{m}}, \tau\right)$ becomes large as the glass transition is approached, as shown in figure 5; this is the microscopic relaxation time scale, often termed $\tau_{\alpha}$ when referring to the final decay of $F(k, \tau)$ [236]. (See section 2.4 for a discussion of DLS and scattering functions.) Roughly, $\tau_{\alpha} \propto a^{2} / D_{\mathrm{L}}$, where $a$ is the particle radius, and so both $\tau_{\alpha}$ and $D_{\mathrm{L}}$ are considered measures of how microscopic dynamics slow near the glass transition.

The question then is how $D_{\mathrm{L}}$ and $\tau_{\alpha}$ depend on $\phi$ [237, 238]. Results from viscometry and DLS studies were reported 


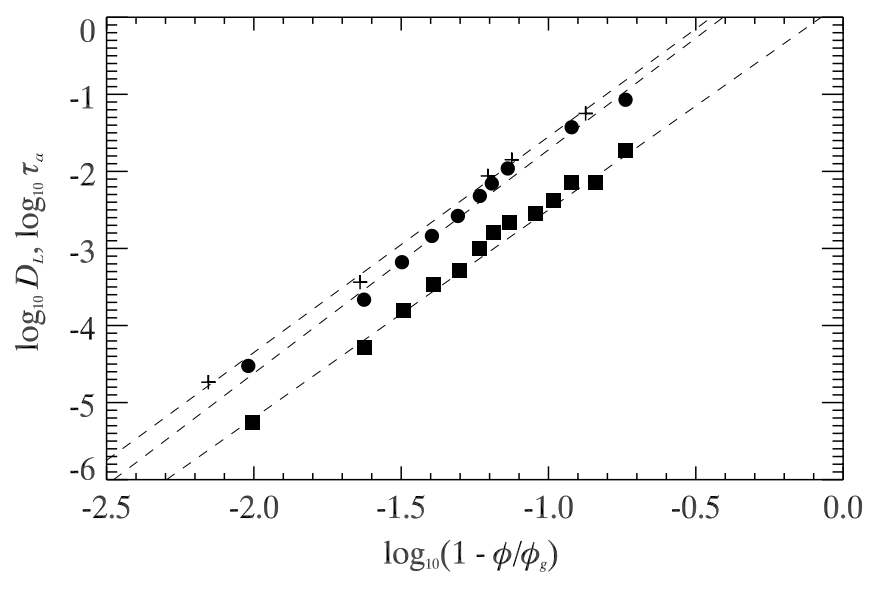

Figure 10. Growing relaxation time scale $\tau_{\alpha}$ (plotted as $-\log \left(\tau_{\alpha}\right)$, circles) and decreasing diffusivity $D_{\mathrm{L}}$ (squares) plotted against the distance to the colloidal glass transition, using $\phi_{\mathrm{g}}=0.571$ for the $D_{\mathrm{L}}$ data and $\phi_{\mathrm{g}}=0.572$ for the $\tau_{\alpha}$ data. Data taken from [100]. Diffusion constants are measured from figure $2(b)$ and $\tau_{\alpha}$ from figure 5. Plus symbols are $\tau_{\alpha}$ data from [247], a prior experiment by the same group.

by Segrè et al for a suspension of PMMA hard spheres over the range of volume fractions $0 \leqslant \phi \leqslant \phi_{\text {freeze }}=0.494$ [239]. Intriguingly, they found that the growth of $\eta(\phi)$ (measured with a rheometer) was well matched by the growth of the inverse diffusion constant, $\left(D_{\mathrm{L}}\left(k_{\mathrm{m}}\right)\right)^{-1}$. This suggests that at least to $\phi \approx 0.494$, viscosity and diffusion are well coupled. It is known that in molecular glasses, these two quantities can decouple: diffusion is quite slow, but not as slow as would be expected from measurements of $\eta$ [236, 240-242]. van Megen et al acquired data up to $\phi_{\mathrm{g}}-0.01$ and found that $D_{\mathrm{L}}$ and $\tau_{\alpha}$ remain well coupled; their data are shown in figure 10 . Results for both $\eta$ and $\tau_{\alpha}$ from the same sample with $\phi>0.494$ have not yet been obtained, partly because of the experimental difficulty of making the different measurements at exactly the same volume fraction [66, 243,244]. Comparing data sets from different groups suggests that perhaps $\eta$ and $\tau_{\alpha}$ remain coupled [245], although such comparisons are non-trivial and the results should be treated cautiously [66]. In general, it is hard to accurately determine how $\eta(\phi)$ grows near $\phi_{\mathrm{g}}$ or $\phi_{\mathrm{rcp}}$, partly because of the difficulty in measuring $\phi$ accurately, and partly because small changes in $\phi$ make a large difference, precisely as shown in figures 8 and 10. It is worth noting that some experimental differences between $\eta(\phi)$ and $D_{\mathrm{L}}(\phi)$ may be due to slip at the particle surface $[239,246]$. As already noted, another difficulty in measuring $\eta(\phi)$ for $\phi>0.5$ arises due to the very slow shear rates required to do so-the need to shear at such low rates is itself evidence for a dramatically growing microscopic relaxation time scale [28].

Given the difficulties of doing both viscometry and DLS on the same samples, and given the power of DLS compared with viscometry, it is natural that many people have used DLS to examine how $\tau_{\alpha}$ grows as $\phi \rightarrow \phi_{\mathrm{g}}$. van Megen's group has performed well-known DLS experiments over three decades starting in the 1980s [32,100,142,247-251]. One of their notable findings is that the increase in $\tau_{\alpha}(\phi)$ is well described by MCT; see, for example, [1,247, 252, 253]. Significantly, not only is $\tau_{\alpha}$ fit by MCT, but several other features of $F(k, \tau)$ are as well, with the only adjustable parameter being the scaling of the volume fraction $\phi$.

The experiments by van Megen et al were performed with hard-sphere-like colloidal particles; parallel experiments were performed by Bartsch, Sillescu and co-workers starting in the 1990s, using softer colloidal particles [104, 105, 254260]. Some intriguing differences were seen; for example, some relaxation processes still appeared to persist in the glass phase [105, 254]. Their early observations suggested a glass transition at $\phi \approx 0.64$ [104], but this was later attributed to depletion effects due to the likely presence of free polymers [256, 258]; see also the discussion in section 5.1. Later experiments by the same group found a glass transition at $\phi_{\mathrm{g}}=$ 0.595 , which was higher than 0.58 likely due to the moderately high polydispersity of these samples $(\sim 16 \%)$ [256]. Many of the predictions of MCT were confirmed in these soft particle experiments [105, 255], suggesting that the particle properties are not crucial.

The hard-sphere results have been recently updated by Brambilla et al who studied samples with $\phi>0.58$ using DLS [261,262]. Amazingly, they found that these slow and dense samples eventually equilibrated. Some of their results agreed quite well with the earlier work of van Megen et al. However, above $\phi_{\mathrm{c}}$, the volume fraction where MCT predicts a divergence of $\tau_{\alpha}$, they instead found finite values of $\tau_{\alpha}$-suggesting that these samples were not yet glasses and that $\phi_{\mathrm{c}}$ of MCT is not equivalent to $\phi_{\mathrm{g}}$ for their samples. These results are controversial [263-266]; key issues raised are difficulties determining volume fractions and difficulties comparing results with differing polydispersity [66].

It should be noted that there are other predictions of how $D_{\mathrm{L}}(\phi)$ should behave near the colloidal glass transition. A notable theory taking into account hydrodynamic interactions is due to Tokuyama and Oppenheim [267, 268], which predicts a glass transition at a specific volume fraction $\phi_{0} \approx 0.5718$ (with an exact expression given for this value).

Some understanding of how structure and dynamics relate to each other has been recently presented by van Megen et al [251, 269]. They studied sterically stabilized PMMA particles in decalin, which were well characterized to behave as hard spheres. In [251], they studied the MSD and identified the time scale $\tau_{\mathrm{m}}^{(\mathrm{s})}$ at which the MSD had the smallest logarithmic slope, that is, the time scale at which the MSD was the most subdiffusive. Here the (s) superscript indicates selfmotion. They also identified an analogous time scale for collective motion, $\tau_{\mathrm{m}}^{(\mathrm{c})}(k)$, directly from DLS data, which was approximately the same as $\tau_{\mathrm{m}}$. These time scales both grow as the glass transition is approached, which makes sense. By examining the $k$ dependence of $\tau_{\mathrm{m}}^{\text {(c) }}(k)$, they showed that structural arrest - the slowing of the motion — starts at length scales corresponding to $1 / k_{\mathrm{m}}$, where $k_{\mathrm{m}}$ is the peak of the static structure factor, and then spreads to other length scales [251]. This suggests that as the glass transition is approached, the spatial modes of motion do not slow uniformly; that some are frozen out sooner. A related study by the same authors found that the dynamics exhibit qualitative changes for the metastable states, that is, $\phi>\phi_{\text {freeze }}=0.494$ [269].

We refer the reader to $[1,72]$ for good reviews of experiments studying diffusion and relaxation times for the 

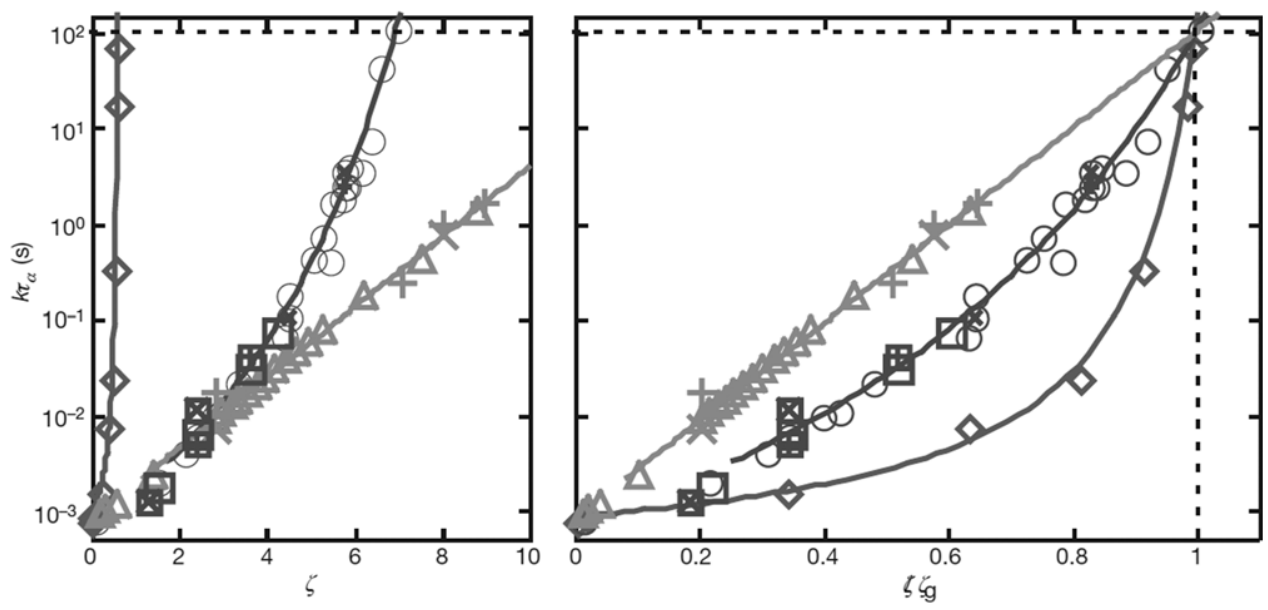

Figure 11. (a) Plot of scaled relaxation time $k \tau_{\alpha}$, where $\tau_{\alpha}$ is measured from light scattering and $k$ is chosen to collapse the data at low $\zeta$ values. Symbols are diamonds (stiff particles), circles and squares (intermediate stiffness), triangles (soft particles), crosses and pluses (rescaled shear viscosities from rheology measurements, corresponding to intermediate and soft particles, respectively). (b) Same data as (a), with the effective volume fraction $\zeta$ normalized by $\zeta_{\mathrm{g}} \equiv \zeta\left(k \tau_{\alpha}=100 \mathrm{~s}\right)$. Reprinted by permission from Macmillan Publishers Ltd: Nature [276], copyright 2009.

colloidal glass transition, and how the experiments relate to MCT predictions. Earlier reviews summarize the state of these questions in 1998 [71] and 2001 [49]. MCT is specifically reviewed in $[2,270,271]$.

\subsection{Fragility}

An important feature of molecular and polymer glasses is that, while the relaxation time scale grows dramatically in all cases, the rate of this growth varies between different samples. This difference is termed the fragility [3]. Fragile glass-formers are ones in which the relaxation time scale grows slowly over some range of the control parameter, and as the glass transition is approached, increases quite suddenly. Such glasses are 'fragile' in the sense that when their viscosity is high, a slight change in the control parameters (increasing $T$ or decreasing $P$ ) results in a sharp decrease in the viscosity, easily 'breaking' the glassy behaviour. In contrast, 'strong' glasses exhibit Arrhenius behaviour, where time scales and viscosity grow smoothly and steadily as the glass transition is approached [6]. Results shown in figure 11(b) are from colloidal glasses and illustrate the types of behaviours one might see: here the straight-line data are from a strong glass, and the curved data correspond to fragile glasses.

The fragility can be defined in several ways. One common way is to fit the viscosity as a function of $T$ to the VogelTammann-Fulcher equation:

$$
\eta / \eta_{0}=\exp \left[D T_{0} /\left(T-T_{0}\right)\right]
$$

Here $\eta_{0}$ is the viscosity at large $T$, and $T_{0}$ results from a fit to where the viscosity would become infinite. The parameter $D$ is called the 'fragility index', and is larger for stronger glasses; $D \rightarrow \infty$ corresponds to Arrhenius behaviour. For fragile polymer glasses, $D$ can be as low as $\sim 2$ [3]. This non-Arrhenius behaviour is taken as a sign that molecules cooperate in their motion; diffusion and structural relaxation are no longer local processes [272]. For colloids, one might exchange $T_{0} / T$ with $\phi / \phi_{0}$, given that for regular glasses one decreases $T$ to $T_{\mathrm{g}}$ and for colloids one increases $\phi$ to $\phi_{\mathrm{g}}$. Thus colloids would be fit with the Doolittle equation (15) with $C=1$ :

$$
\eta / \eta_{0}=\exp \left[D \phi /\left(\phi_{0}-\phi\right)\right]
$$

Using this definition, hard-sphere colloids appear to be fragile glass-formers with $D \approx 1.15$ (from figure 8 [221]) or $D \approx 1.65$ (from rheology [28]). Equivalent formulas can be written using $\tau_{\alpha} / \tau_{0}$; light scattering data on hard-spherelike colloids suggest $D \approx 0.50[202,261,262]$. However, the exchange of $T_{0} / T$ with $\phi / \phi_{0}$ is perhaps misleading [272]. In [272], Berthier and Witten argue that $\phi$ is more analogous to energy density, and that the non-dimensional pressure $1 / Z=$ $P V_{\mathrm{p}} / \phi k_{\mathrm{B}} T$ is more analogous to $1 / T$, using pressure $P$ and particle volume $V_{\mathrm{p}}$. As $P$ has a non-trivial $\phi$ dependence, they point out that the relationship between $\eta$ and $\phi$ can be confusing when comparing different types of colloidal particles, especially as $Z$ is generally not easily measurable in an experiment. Overall, however fragility might be defined, an important question is 'what features of a glass-forming material relate to the fragility?' [202, 273-275].

This question has recently been explored by Mattsson et al [276] with colloidal suspensions of soft hydrogel particles. These particles easily deform and so can be compressed as their concentration is increased; for this reason, their glass transition does not occur at the same volume fraction of $\phi_{\mathrm{g}} \approx 0.58$ as for hard particles. The authors considered a generalized volume fraction $\zeta=n V_{0}$, where $n$ is the particle concentration and $V_{0}$ is the volume of an undeformed particle. Given that particles can be compressed to much less than $V_{0}$, the generalized volume fraction $\zeta$ can greatly exceed 1. Shown in figure 11, Mattsson et al found that softer particles (triangles) behaved as strong glasses, while harder particles (circles, diamonds) behaved as more fragile glasses, using the equivalence between $1 / T$ and $\zeta$. This is an exciting demonstration of a model colloidal system which 
can be used to explore fragility. However, an open question is whether using the non-dimensional pressure $Z$ would perhaps collapse the data between the differing particle types [272]. The simulations of [272] show that hard spheres are 'fragile' when plotted against $Z$.

The results of Mattsson et al are interesting, but it is not completely clear how particle softness relates to fragility. A simulation of different particle potentials did not find any fragility changes [277], although this study only considered varying $T$ rather than density. Certainly for polymer glasses, fragility can be quite different depending on if $T$ or density is varied [275]. Furthermore, 'softness' has two distinct meanings. In simulations such as [277], softness refers to the shape of the interparticle potential. Often the repulsive part of the potential decays as $1 / r^{n}$, and smaller values of $n$ are considered softer particles. In contrast, Mattsson et al used softness to refer to the particle modulus [276], which probably is a prefactor to an interparticle potential with fixed shape.

A recent mode-coupling study suggests that both the shape of the potentials and their prefactors may be needed to understand Mattsson et al's results [278]. For example, if the interparticle potential is $\sim 1 / r^{2}$ at large separations $r$, and $\sim 1 / r^{6}$ at smaller separations, then increasing particle concentration can shift the scale of the interaction energy to a different regime of the potential. If such an effect was properly accounted for, all of the data might collapse for different softnesses [59]. In a sense, this suggests that the results for the soft spheres can be considered in terms of their effective hard-sphere size $[279,280]$, thus explaining the results in terms of a mapping from $\zeta$ to $\phi_{\text {effective }}[59,281,282]$. However, the functional form of the interparticle potential is unknown for hydrogel particles. Furthermore, it is not known if or how the interparticle interactions vary between different batches of Mattsson et al's particles. (Other groups have noted that their particles vary from batch to batch: see the discussion in [282] comparing their results with their prior work in [283].) While the results of Mattsson et al are exciting, they raise many questions. A full understanding requires either precise knowledge of how hydrogel particles interact or insights from simulations on how to replicate the experimental data.

One can also consider the results of Mattsson et al in another way. The Arrhenius $\zeta$-dependence for the softer particles suggests that the energy barrier for rearrangements is independent of $\zeta$. Perhaps soft particles can rearrange without significantly affecting others, and so rearrangements involve only a few particles. In contrast the harder particles might exhibit growing dynamical heterogeneity (see section 3.3), requiring more and more particles to rearrange, and thus leading to a growing energy barrier with $\zeta$. If these conjectures are true, this would suggest that soft particles are not effectively hard particles with a different radius. Microscopy experiments may be able to shed light on the question of particle rearrangements.

Recent simulations suggest different ways to tune the fragility of colloidal systems. One simulation showed that fragility was tunable using a binary system and controlling the size ratio and number ratio of the two species [202]. While intriguing and potentially useful for tuning the properties of colloidal suspensions, a binary system would be of limited use for understanding the fragility of single-component molecular glasses. Another simulation studied soft particles with finiterange potentials, quite analogous to soft colloidal particles, and found that the temperature-dependent fragility increased dramatically when the particles were over-compressed (density increased above the point where the particles had to interact) $[272,280]$.

\subsection{Dynamical heterogeneity}

In a liquid below its melting point, some regions may exhibit faster dynamics than others even though, spatially, these regions may be very close [236, 284,285]. This behaviour is called dynamical heterogeneity, and illustrates that different regions of a system relax at different rates. In such a system, relaxation time scales and length scales are coupled, that is, longer relaxation times are typically associated with larger collections of particles. One key idea here is that of cooperative motions [286]: near the glass transition, perhaps molecules need to 'cooperate' in order to rearrange.

Cooperative motion has been seen in colloidal samples, such as the one shown in figure 12. The left image shows a raw confocal microscope image, a 2D slice through a 3D sample at $\phi=0.46$. The right image shows the difference between particle positions in the left image and an image taken $60 \mathrm{~s}$ later. Some regions of this image are grey, indicating places where particles move relatively little during this period of time. Other regions are black and white, indicating groups of particles all moving together. For these groups, motion is from black to white; for example, the anomalously large particle moves slightly to the left. It can be seen that in general, neighbouring particles that are rearranging tend to move in similar directions. This sample is relatively far from the glass transition (the images come from a sample with $\phi=0.46$ as compared with $\phi_{\mathrm{g}}=0.58$ for these experiments). As the glass transition is approached, the regions of rearranging particles grow larger, but the displacements during a given rearrangement are smaller [137], so similar images at larger $\phi$ are less revealing. Overall, observations of spatially heterogeneous motion have been seen with microscopy in several colloidal experiments [110, 112, 128, 129, 287-289].

The interpretation of these results relate to cage trapping and cage rearrangements. As described in section 1.5, at short times, particles move due to Brownian motion, but this motion is constrained because particles collide with their neighbours. The neighbours thus 'cage' the particle-of course, the particle is also part of the cage around its neighbours [2,93-98]. On longer time scales, the cages relax and the system rearranges. As figure 12 shows, rearrangements often occur when one particle moves, another particle follows, and so on.

As the glass transition is approached, the size of the cooperative groups of particles increases, as well as the time scale for these motions [99, 128, 129, 137, 290]. The growing length scales (quantified from spatial correlation functions) extend up to $\sim 4-5$ particle diameters [198, 290], shown by circles in figure 13. Rearrangements can involve up to $\sim 200$ particles [129], and the average size of a rearranging region 


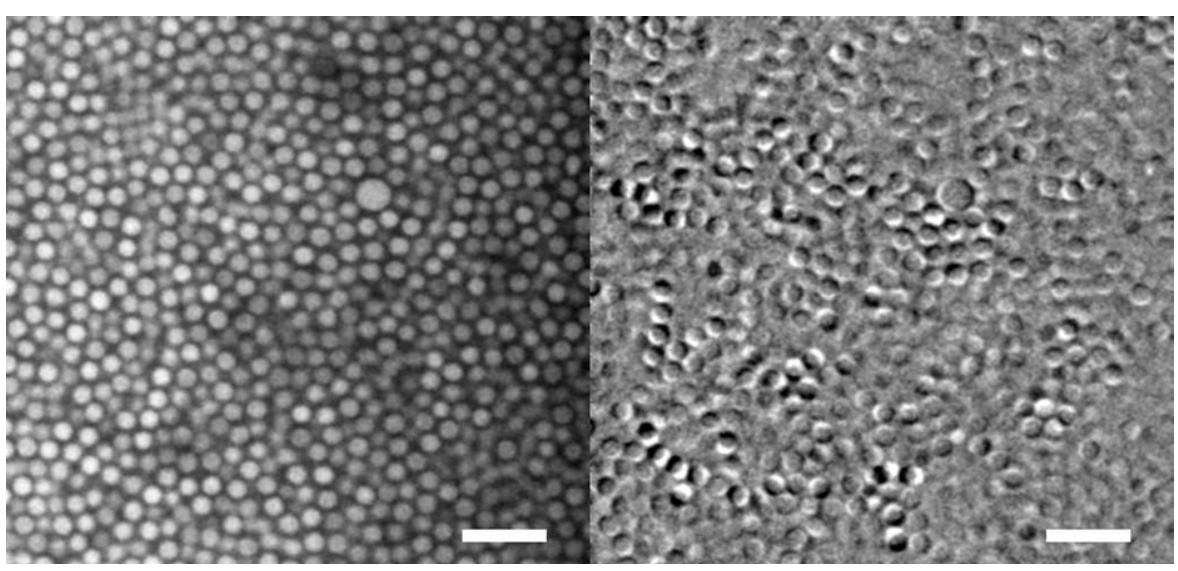

Figure 12. Left: confocal microscope image of a sample with $\phi=0.46$. Right: difference between the left image and one taken $60 \mathrm{~s}$ later. Where this image is grey, nothing has moved; where it is black, a particle existed at the earlier time, and where it is white, a particle existed at the later time. Of course, particle motion also occurs perpendicular to the plane of the image. In both images, the scale bar indicates $10 \mu \mathrm{m}$. For this sample, the particles are slightly charged, shifting the onset of freezing from $\phi_{\text {freeze }}=0.494$ to $\approx 0.42$ [48].

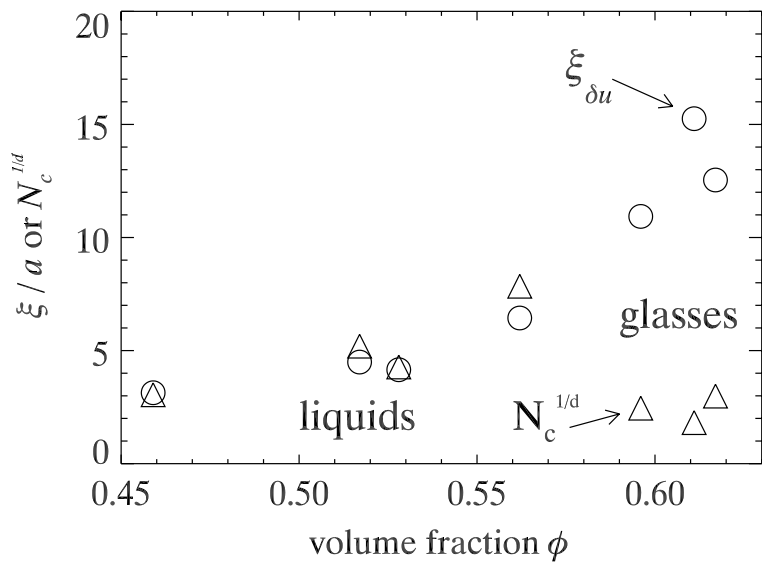

Figure 13. Data on growing length scales for dynamical heterogeneity in colloidal samples. Circles: length scales $\xi_{\delta u}$ found from spatial correlations of mobility, normalized by particle radius $a=1.18 \mu \mathrm{m}$ [290]. Triangles: mean cluster size in terms of number of particles $N_{\mathrm{c}}$, converted to a length scale using the fractal dimension $d=1.9$ [129]. These are clusters of mobile particles as defined in [129], and by computing $N_{\mathrm{c}}^{1 / d}$ we find the typical length scale of such clusters. The same data were analysed in $[129,290]$. For these experiments, the glass transition was identified as $\phi=0.58$.

is indicated by triangles in figure 13. Intriguingly, for liquid samples $(\phi<0.58)$, these two length scales are essentially identical. For glassy samples, the correlation length scale is large, but cluster sizes are small. This is probably due to difficulty defining cluster sizes in glassy samples as discussed in [291]. Rearranging particle displacements are small and can be lost in the 'noise' of particles diffusing within their cages in a glassy sample. More careful analysis reveals larger clusters in glassy samples, although the details of defining such clusters are ambiguous in glasses due to ageing [291] — see the discussion in section 4.2.

These experimental observations of spatial dynamical heterogeneity are in good agreement with prior observations in computer simulations [56, 194-196, 198-200, 204, 292]. While the increasing size of these cooperative regions is a striking observation, it is a bit unclear exactly how this relates to the growing relaxation time [197,293]—is it a cause, effect, or side-effect? Intuitively, it makes sense that if more and more particles need to move simultaneously in some coordinated fashion, that this is hard to do and will occur less often, thus connecting directly to slowing time scales for diffusion. In this sense, dynamical heterogeneity and the glass transition are strongly connected, and the former could be said to 'cause' the latter. Some evidence for this comes from simulations which see diverging measures of dynamical heterogeneity [196, 292, 294,295]. One intriguing recent result comes from simulations of a 4D hard-sphere system: there a glass transition was seen, but dynamical heterogeneity was much less significant [208]. This suggests that perhaps glassy behaviour can occur for other reasons. (As noted in section 2.6, in general similar phenomena are seen in two dimensions and three dimensions, and one would assume that a $4 \mathrm{D}$ simulation can still provide useful insight into the $3 \mathrm{D}$ problem.)

It would be useful to understand the factors that allow some particles to rearrange, or conversely the factors that prevent the other particles from doing so. It has been noted that higher local volume fractions are weakly correlated with reduced mobility $[137,296,297]$. The correlation is sufficiently weak that it has essentially no predictive ability. Simulations, though, have shed some light on this. Widmer-Cooper et al conducted simulations that showed some regions within a sample have a higher 'propensity' to rearrange [203,216]. To come to this conclusion, they ran repeated simulations beginning with the same initial particle configurations. From the initial configuration, the system was evolved using molecular dynamics and randomizing the velocities of the particles. Although again, these sites were only weakly correlated with structural properties [203,216,298,299]. Such a procedure has not yet been tested in a colloidal experiment. Investigating dynamical heterogeneity is a good example where simulations are quite powerful; simulations preceded and guided the experimental data analysis [204], and allow for studies that are experimentally difficult or even impossible [203, 208].

Figure 12 shows clear spatial variations in mobility. However, another mode of studying dynamical heterogeneity 
is to consider the temporal fluctuations of mobility: in any given region, the amount of motion will fluctuate in time $[67,291,300]$. These fluctuations can be quantified with a four-point susceptibility function called $\chi_{4}$ [294,295,301303]. This function has been shown to be closely related to the cooperative motion discussed above, and can be used to pick out a time scale corresponding to the dynamical heterogeneity. The analysis has been successfully applied to colloidal experiments several times [301,304-306], with the results essentially in agreement with what has been seen in simulations.

One could argue that studies of glassy systems should focus on understanding the behaviour of slow particles, rather than fast ones. After all, over a given period of time the overwhelming majority of particles in a glassy system are fairly immobile. By studying mobile particles, one learns how mobility decreases as the glass transition is approachedfor example, particle motions are not only rarer, but also smaller [137]. Also, it is usually the case in experiments that faster moving particles are easier to distinguish. However, one confocal microscopy study focused its analysis on less mobile particles [167], finding that clusters of slow particles percolate through a colloidal glass.

Dynamical heterogeneity in molecular glass experiments is reviewed in [236, 284, 285]. Additionally, a chapter in [162] gives a lengthy review of dynamical heterogeneity in colloidal glasses. An earlier review of dynamical heterogeneity in soft glassy materials is [151].

\subsection{Confinement effects}

Phase transitions are usually investigated in the context of macroscopically large systems. However, confining samples so that one or more dimensions are microscopic leads to new physics, including confinement-driven phases [307]. For amorphous phases, the glass transition temperature $T_{\mathrm{g}}$ is often changed by confinement [308-318]. In some experiments, the glass transition temperature is decreased upon confinement (as compared with the transition temperature in bulk) $[57,209$, $312,313]$, whereas in others, the glass transition temperature increases [209, 308,310,319]. In some cases, $T_{\mathrm{g}}$ can increase or decrease for the same material, depending on the experiment [209, 308, 313, 317]; this is likely due to differing boundary conditions [308]. In molecular glass experiments, important differences are found when studying confined samples supported by substrates, as compared with freestanding films [308,315-317]. In other experiments, results depend on whether the confining surface is hydrophobic or hydrophilic. Computer simulations indicate that confinement influences the arrangement of atoms [57, 209, 210,320], which might in turn relate to the change in the glass transition temperature. However, it is difficult to probe the structure and dynamics of nano-confined materials.

Colloids thus can serve as an excellent model system for studying confinement effects. Such experiments have been performed by two groups who confined samples between parallel glass plates [213,321]. Nugent et al used a binary sample to prevent crystallization [213], while Sarangapani and

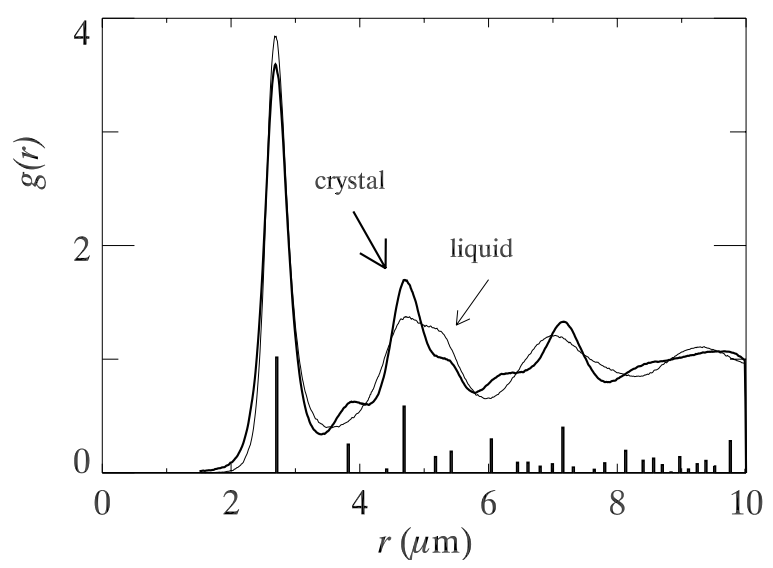

Figure 14. Pair correlation function $g(r)$ for a liquid sample $(\phi=0.48)$ and a crystalline sample $(\phi=0.57)$, with particle radius $a=1.18 \mu \mathrm{m}$. The particles are the same colloidal PMMA spheres used in [129], and were observed using confocal microscopy. For perfectly hard particles, the position of the first peak in $g(r)$ would be at $2 a$. Here, however, the position of the first peak is at $\sim 2.7 \mu \mathrm{m}$, larger than $2 a=2.36 \mu \mathrm{m}$, because the particles are slightly charged. The vertical bars at the bottom edge of the graph are the positions of the peaks of $g(r)$ for an ideal random hexagonal close-packed crystal. Properly speaking, these should all be Dirac delta functions (infinitely high); here they are truncated by finite resolution, and rescaled but still proportional to their magnitude in the ideal case. The peaks of the experimental crystal are broadened due to Brownian motion around the particles' lattice sites, a slight polydispersity and also particle-tracking uncertainty.

Zhu studied a monodisperse sample [321]. Both experiments used confocal microscopy to observe a dramatic slowing down of particle motion in samples that were very confined. This suggests that the glass plates act analogously to 'sticky' boundaries in the molecular glass experiments conducted on substrates, which also find a slowing down of particle motion [308]. Follow-up work showed that rough confining surfaces slowed motions even further [215]. The experiments show a clear connection between layering of particles against the walls and their mobility [213], which has also been studied by simulation [57, 322, 323].

\section{Features of glassy systems}

\subsection{Amorphous solids}

It is visually apparent from the bottom of figure 1 that colloidal glasses and crystals have different structures. Repeating patterns, like those in a crystal, are completely absent in the glassy state and instead the glass more closely resembles a very dense liquid. Although liquid-like, the system is dense enough that it can bear some degree of stress over short time periods and respond elastically (see $G^{\prime}$ in figure $7(a)$ ). Thus, glassy systems are commonly described as amorphous solids.

A simple measure of structure is the pair correlation function (or radial distribution function), $g(r)$, which describes fluctuations in particle number density at a distance $r$ away from a given particle. The pair correlation functions for a colloidal crystal and a colloidal liquid are shown in figure 14. The curve for the crystal (bold line) has fluctuations at definite positions, corresponding to spacings between particles in 
the random hexagonal close-packed lattice. If this were an ideal crystal, these fluctuations would be narrow spikes, but in figure 14 they are broader due to Brownian motion, polydispersity, and particle-tracking uncertainties, all of which ensure that particle positions are not on exact lattice sites. The liquid curve (thin line in figure 14) differs significantly from the crystal, reflecting that the sample itself has much less structure. The second peak for the liquid, around $4<r<6 \mu \mathrm{m}$, is slightly split into two sub-peaks. As can be seen, these correspond to two features of the crystal curve. The origin of the sub-peaks is local packings of three or four particles that appear crystalline and result in spacings of second-nearestneighbour particles that correspond to the crystalline spacings. In some experiments, this split second peak of $g(r)$ is more obvious, and sometimes taken as a signature of the glassy state; however, this is not a defining feature but merely a common observation.

In 1991, Snook et al used SLS to study the structure of colloidal glass samples [54]. Comparison with simulations of random close-packed spheres confirmed that the experimentally obtained colloidal glass was indeed amorphous. Later in 1995, van Blaaderen and Wiltzius used confocal microscopy to examine the amorphous structure of a colloidal glass [127]. They studied quantities such as the number of nearest neighbours, bond-order parameters, and $g(r)$, and found that all were in good agreement with simulations of random close packing [127]. More recently in 2010, Kurita and Weeks used sedimentation to obtain a random close-packed sample and imaged nearly half a million particles (using confocal microscopy), again finding that the experimental sample was quite similar in many respects to simulations of random close packing [324]. Their large sample size enabled a comparison with recent simulations which found 'hyperuniformity,' meaning that density fluctuations disappear linearly with wavelength in the long wavelength limit [325]. An implication of a sample being hyperuniform is that it is incompressible, which has been conjectured to be a requirement for random close-packed systems [326]. The only disagreement with simulations was that the experimental density fluctuations did not go to zero at long wavelengths, implying that the sample was compressible. However, recent simulation results suggest that the disagreement is due to polydispersity in the experimental sample [327-329]. Reanalysis of the Kurita and Weeks data found that accounting for polydispersity does indeed demonstrate that the experimental samples are hyperuniform, and thus incompressible [330]. However, liquids and glasses have a finite compressibility: these observations of incompressibility in random closepacked samples, then, demonstrate a structural difference between such samples and liquids and glasses. This shows the limits of such samples as models for liquids, which is intriguing given that random close-packed hard spheres are one of the original models for liquids [34].

\subsection{Ageing}

As with their molecular and polymer counterparts, colloidal glasses exhibit ageing - as the system evolves towards

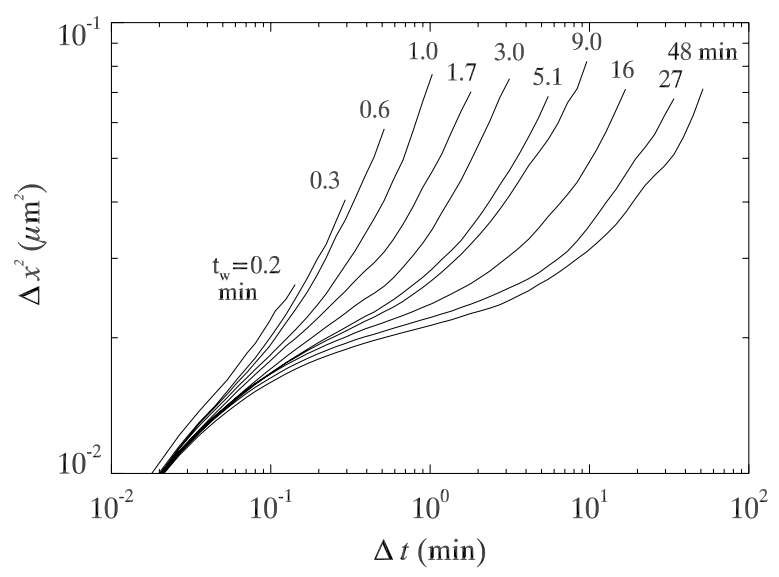

Figure 15. The MSD for an ageing colloidal sample. The waiting time is labelled for each curve. Data from [291], corresponding to a sample with $\phi \approx 0.62$ observed with confocal microscopy. Note that each curve is averaged over a window of time centred on the $t_{\mathrm{w}}$ indicated; see [291] for details of this window averaging. The curves are evenly spaced in $\log \left(t_{\mathrm{w}}\right)$, but not evenly spaced on the graph, reflecting the fact that within the imaged region $(\sim 1000$ particles), ageing takes place intermittently [300,340].

equilibrium, measured properties may change with time [80,164,331-335]. In a sense, ageing can be thought of as a transient effect: consider a supercooled fluid whose temperature is decreased slightly from $T_{1}$ to $T_{2}$. If the relaxation time scale at $T_{2}$ is $\tau_{2}$, then equilibration to the new temperature occurs over a period $\sim \tau_{2}$ [336]. During the equilibration process, the dynamics depend on the waiting time $t_{\mathrm{w}}$ since the temperature was changed to $T_{2}$. These $t_{\mathrm{w}}$ dependent dynamics are ageing, and if $\tau_{2}$ is sufficiently large, then the sample can be considered a glass which will age for as long as an experiment is performed [337]. In contrast, for supercooled liquids the same ageing phenomena are seen, but only for $t_{\mathrm{w}} \lesssim \tau_{2}$; here, the phenomena might equally well be described with the word 'equilibrating' [80].

Ageing is readily observed in colloids by examining the MSD at different waiting times, $t_{\mathrm{w}}$, where the waiting time is defined as the time since the last perturbation. The system in figure 15 displays a slowing of dynamics as $t_{\mathrm{w}}$ is increased (with $t_{\mathrm{w}}=0$ defined as the end of an initial stirring, see below) [291]. The short time dynamics are unchanged and reflect particles diffusing within their cages. But with increasing $t_{\mathrm{w}}$, the plateau broadens and the upturn occurs at longer $\Delta t$, indicating that relaxation occurs over increasingly larger time scales. Analysis of these data found that particle mobility was related to $\log \left(t_{\mathrm{w}}\right)$ [338]. Analysis of light scattering data, in contrast, found time scales for motion which depended algebraically on $t_{\mathrm{w}}$ [335]. For all of these experiments, ageing occurs over the entire duration of the experiment, and so these samples are easily classified as a glass. There is recent evidence, though, that even fairly dense colloidal samples can eventually equilibrate given enough time, raising questions about how to best define $\phi_{\mathrm{g}}[261,339]$.

In molecular or polymer glasses, vitrification occurs when the temperature is quickly lowered, namely the system is thermally quenched. In colloids, the creation of glassy systems typically involves slow centrifugation of a sample. Hence, the 
time at which the system becomes glassy is poorly defined. To this end, the study of colloidal glasses often begins with a process known as shear rejuvenation in which the system is stirred or sheared in order to remove any history dependence [341-345]. The hope is that vigorous stirring breaks up any subtle structure in the sample so that experiments begin with a randomized initial structure. The time that the stirring stops defines $t_{\mathrm{w}}=0$ [146]. Subsequent ageing, then, is presumably a slow evolution of the structure to some 'older' state [346]. There is evidence that in some cases the shear rejuvenation process produces different types of behaviour than those observed in polymer glasses that are thermally quenched [345, 347, 348].

A process more analogous to a thermal quench would be to increase the volume fraction from $\phi_{1}$ to $\phi_{2}>\phi_{1}$. This is possible with hydrogel particles, whose size is controllable by temperature (see section 5.1). To date, there have been several important ageing experiments using these particles. Purmono et al studied the rheological behaviour of ageing hydrogel samples [345,349]. They found that both shear rejuvenation and changing the volume fraction resulted in reproducible initial states, although they were slightly different. Subsequent microscopy experiments by the same group used tracer particles to confirm and extend these results [350]; in particular, the sample was revealed to be spatially dynamically heterogeneous, with mobile and immobile particles coexisting. Other experiments by this group demonstrated that ageing behaviour was independent of particle softness, despite the glass transition depending on both volume fraction and particle softness [351]. In another experiment, Yunker et al studied dynamical heterogeneity during the ageing of a quasi-2D sample and found that the size of rearranging regions increased as the sample grew older [111]. These results contrast with earlier work done in three dimensions with a shear rejuvenated sample [291]; differences in dimensionality and quench method are both plausible explanations for the different observations. In another hydrogel experiment, Di et al [352] replicated certain classic ageing experiments [353,354]. For example, in 1964 Kovacs found that molecular glasses quenched to $T_{2}$ from $T_{1}>T_{2}$ would approach equilibrium differently than if heated to $T_{2}$ from $T_{3}<T_{2}$. Di et al observed the same 'asymmetric approach' using hydrogel particles observed with DWS (see section 2.4).

A natural question to ask is 'are there structural signatures of ageing?' That is, given information about the structure of two samples, is there some quantity that distinguishes an 'old' sample from a 'young' one? Indeed, it is somewhat intuitive to expect a correlation between ageing dynamics and structure. In polymer glasses, for example, systems become denser as they age [355]. For a colloidal glass composed of hard-sphere-like particles, presumably the only 'clock' in the sample is the structure; the sample should have no other way of knowing $t_{\mathrm{w}}$. The relevant quantity in colloidal glasses, however, has remained elusive. Cianci et al $[346,356]$ searched for correlations between the structure and dynamics of an ageing colloidal glass, specifically looking at four-particle tetrahedral configurations within the system. A wide range of structural parameters were examined but none changed with $t_{\mathrm{w}}$. However, ageing did occur intermittently [300,357] via spatially localized groups of particles [146, 291], similar to what has been seen in simulations [334, 358-360]. These slightly more mobile particles were associated with more disordered local environments [346]. Presumably the more mobile particles are the ones that change the structure and thus are responsible for the sample having a larger age, but the connection between structure, dynamics and age remains unclear [346]. A similar set of observations was found by the same group studying a binary sample: local composition, quantified by relative numbers of small and large particles, influenced dynamics but did not itself change as the sample aged [146]. These colloidal observations are in reasonable agreement with simulations, which found that structural changes during ageing are quite subtle [333,361]; they also agree correlations between structure and mobility in other simulations and experiments [137, 362, 363].

Ageing has also been considered in regard to the fluctuation-dissipation theorem [364], which connects temperature, viscous friction and interparticle potential to diffusive motion. In the absence of interparticle potentials, as in the case of hard-sphere particles, fluctuation-dissipation can be written simply as

$$
D=\frac{k_{\mathrm{B}} T}{C},
$$

where $C$ is a viscous drag coefficient. The drag force on a sphere moving at velocity $v$ is $F_{\text {drag }}=C v$ (see (8)). Therefore, by measuring the velocity $v$ of a sphere feeling a known external force, and measuring $D$ for the same sphere with no external force, one can calculate $T$. In the same way that MSD has a non-trivial dependence on lag time $\Delta t$ and waiting time $t_{\mathrm{w}}$ (as shown in figure 15), so $D$ can be considered to be a function of frequency $(\omega \approx 2 \pi / \Delta t)$ and $t_{\mathrm{w}}$, and likewise the relationship between $F_{\mathrm{drag}}$ and $v$ may have non-trivial frequency dependence [174]. The ratio of these quantities can be generalized to provide an effective temperature, $T_{\text {eff }}$ [364]. This idea has been tested in a wide variety of non-equilibrium situations, including sedimentation experiments [365], simulations of a sheared foam [366], granular experiments [367,368], simulations of glasses [334, 369], experiments with regular glasses [370, 371] and experiments with colloidal glasses [245, 357, 372-375]. For hard-sphere colloidal glasses, the calculated effective temperature is a few times greater than the actual temperature [245, 374]. Although this may seem counter-intuitive, the observation can be rationalized by arguments from statistical mechanics. Temperature is essentially a measurement of the width of the distribution of energy states. One can think of particle rearrangements as the exploration of various energetic configurations available to the system: for low to moderate $\phi$, rearrangements are easy and the fluctuations in energy from one configuration to the next are small; for $\phi \approx \phi_{\mathrm{g}}$, rearrangements require cooperative motion between many particles and therefore larger energy fluctuations. As the energy distribution is broader for $\phi \approx \phi_{\mathrm{g}}$, this automatically implies a higher effective temperature. Thus, the measured 
$T_{\text {eff }}$ is related to cooperative motion and large scale structural rearrangements within the glass [374]. Although the effective temperature is larger than $T$, it was also found that $T_{\text {eff }}$ does not seem to change with age [374].

Recent experiments with magnetic particles [376] allow the possibility of doing experiments with an ultrafast effective concentration quench. These particles have a repulsion that is controllable with an external magnetic field, and so their effective size can be rapidly varied. This enables experiments to study ageing at extremely short time scales after the quench.

\subsection{Shear of colloidal glasses}

So far, this review has discussed equilibrium properties of supercooled colloidal fluids and out-of-equilibrium ageing in colloidal glasses. Another way to push a sample out of equilibrium is to apply shear, for example using a rheometer (see section 2.5). The interplay of ageing and shear have been studied by several groups [377-379], and as discussed in section 4.2, shear is often used by colloidal scientists to 'rejuvenate' an ageing sample [291,341-343]. In this section, we will briefly summarize some of the key results that have been found when shear is applied to colloidal liquids and glasses.

One question to be asked is 'how quickly is the sample sheared?' This is quantified by the non-dimensional Pèclet number [380]. This number can be thought of as the ratio of two time scales. The first is the time scale $\tau_{\mathrm{D}}$ a particle takes to diffuse its own radius $a$, defined in (5). The second time scale is the inverse of the strain rate, $\tau_{\mathrm{S}}=(\dot{\gamma})^{-1}$, which is the time it takes for a particle to be sheared a distance equal to its own radius, relative to another particle in an adjacent shear layer. Thus the Pèclet number is given by

$$
P e=\frac{\tau_{\mathrm{D}}}{\tau_{\mathrm{S}}}=\frac{\pi \dot{\gamma} \eta a^{3}}{k_{\mathrm{B}} T} .
$$

This assumes that diffusion is well described by the physics in section 1.5 (see (3)-(5)). More relevantly for colloidal glasses, the long-time diffusion sets the time scale $[174,380]$ : in other words, the time a particle takes to diffuse a distance $a$ is much slower than in a dilute sample. In this case, the modified Pèclet number can be defined in terms of the long-time diffusion constant $D_{\mathrm{L}}$ as

$$
P e^{*}=\frac{\dot{\gamma} a^{2}}{6 D_{\mathrm{L}}}
$$

(compare with (5)). If $P e^{*}<1$, then diffusion is more important for rearranging particles, whereas if $P e^{*}>1$, then shear rearranges particles before they can diffuse an appreciable distance. Thus, if one intends to understand shearinduced behaviours, it is necessary to shear quickly (high $P e^{*}$ ) so that diffusion has insufficient time to equilibrate the system. In contrast, the measurements of $\eta(\phi)$ in section 3.1 wish to avoid shear-induced effects altogether, and so samples are sheared extremely slowly (low $P e^{*}$ ).

The other question is how large a strain should be studied. For small strains, the rheological behaviour is linear [163]; that is, the viscoelastic moduli do not depend on the strain

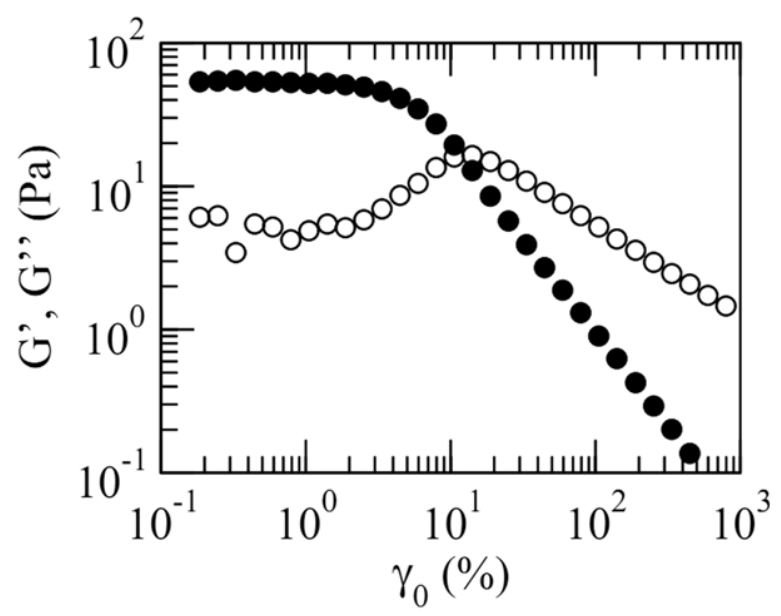

Figure 16. Elastic modulus ( $G^{\prime}$-filled symbols) and loss modulus $\left(G^{\prime \prime}\right.$ - open symbols) as a function of strain for a colloidal glass with $\phi=0.645$. The sample here has a polydispersity of $\approx 12 \%$, hence the large $\phi$. Squares correspond to a slow strain rate $\left(f=1 \mathrm{rad} \mathrm{s}^{-1}\right)$ and circles a high strain rate $\left(f=100 \mathrm{rad} \mathrm{s}^{-1}\right)$. Reprinted with permission from [381].

amplitude (see section 2.5). Early measurements, such as those in figure 16, found that only above a critical strain $\gamma_{\mathrm{c}} \approx 15 \%$ does a colloidal glass sample show significant plastic rearrangements [381,382] (a later experiment using confocal microscopy lowered this to $\gamma_{\mathrm{c}} \approx 8 \%$ [383]). This result supports the idea that a colloidal glass has a yield stress, that is, it is a solid. The elastic response of the sample strained less than $\gamma_{\mathrm{c}}$ has been attributed to a distortion of the nearestneighbour cages surrounding particles (see section 3.3 for a discussion of cages). Equivalently, it can be thought of in terms of free energy; in an unstrained sample, particles are packed randomly in some structure that maximizes entropy and minimizes free energy. When strained, particles move away from that structure at a cost to free energy. So for small strains, the sample gives a linear, elastic response. Above $\gamma_{\mathrm{c}}$, particles break free of their cages and can rearrange [166, 189,380382]. (Although, it is possible that a large region can shift without particles being uncaged in the interior [384].) To some extent, particle rearrangements occur for smaller strains, but may be thermally activated in those cases [385].

Several works have noted that strained systems show dynamically heterogeneity, similar to the thermally activated rearrangements in supercooled colloidal liquids discussed in section 3.3. In other words, the particles that undergo plastic rearrangements in a sheared sample are distributed nonuniformly in space. This has been studied in experiments [380, 383, 385-389] and simulations [201, 388, 390, 391], and was originally predicted by theory [392]. One question that has been raised from studying the shapes of these regions of rearranging particles is 'are these rearranging regions oriented in any particular fashion relative to the shear?' A sheared sample has three unique directions: the velocity direction; velocity gradient direction; and vorticity direction (perpendicular to the first two). Particle motions differ trivially along the velocity gradient direction, and these overall affine motions can be subtracted from the observed particle motion in a confocal microscopy experiment $[139,387]$. Some confocal 
microscopy observations found that diffusion is isotropic $[380,387]$ and that the shapes of the rearranging regions are likewise isotropic [380]. However, simulations suggest that particle motions and rearrangements are not isotropic when sheared in the nonlinear regime [391]. Recent experiments confirm that with the right analysis techniques, anisotropic rearrangements are seen, with long-range correlations due to elastic effects [389]. In general, there are a variety of ways to define and identify plastic rearrangements in a sheared sample; these definitions are compared and contrasted in [380]. The sizes of the dynamically heterogeneous regions appear to be smaller in a sheared sample as compared with the unsheared sample [201,388].

On a more macroscopic scale, a manifestation of spatially heterogeneous motion is shear banding [393-395]. Often when shearing complex fluids, the majority of the strain occurs within a narrow band of the sample, with the rest of the sample remaining relatively unstrained [393-398]. Hence, a measurement of viscosity which assumes uniform flow will lead to incorrect results. Shear banding has been observed and studied in dense colloidal suspensions in recent years [378380, 389, 399-401] and presented problems for rheology for much longer [188]. The origins of shear banding in complex fluids continue to be an active research problem [402].

Given the industrial importance of shearing dense complex fluids, it is unsurprising that the shear behaviour of many different types of complex fluid glasses has been studied. Examples include emulsions [403], soft colloids [404], star polymers [405], colloidal gels [406] and attractive colloidal glasses [407] (see section 5 for the distinction between a colloidal gel and an attractive colloidal glass [408]).

There has also been a fair bit of theoretical work studying the flow of glasses and glassy complex fluids [222, 392, 409412]. Some of these are MCT analyses, which do a good job describing shear of dense colloidal suspensions near the glass transition [410,222]. Other theoretical work examines the flow of polydisperse glassy complex fluids [411,412]. A key prediction of this theory is that a sample will initially build up stress elastically. After some time, a local plastic event occurs and the stress is redistributed in a non-local fashion around the location of the plastic rearrangement. Thus the ability of a local region of the sample to flow depends on what is happening in adjacent regions. This may potentially explain shear banding [412] and other observations of flow profiles in a variety of complex fluids [411,413,414].

\section{Other soft glassy materials}

So far, this review has focused on the colloidal glass transition and experimental work on hard-sphere-like colloidal particles, such as those used in the earliest experiments [30, 142, 248]. We briefly mention here other soft materials that are used for glass transition studies.

\subsection{Soft colloids, sticky particles, emulsions and foams}

Several groups study colloidal glasses composed of polymer hydrogel particles, which interact via a soft repulsion $[104,105,111,223,254-256,258,276,281$, 282, 352,
415-417]. These particles can be packed to volume fractions above $\phi_{\text {rcp }}$ of hard spheres, with potentially interesting consequences (see section 3.2). The stiffness of the particles can be controlled by the amount of cross-linking or using hard cores with hydrogel shells. The size of these particles can be varied by controlling the temperature or $\mathrm{pH}$ [418]. Compressed particles are harder, while swollen particles are softer: this allows for the same particles to be used to test how particle softness influences behaviour [276] and to look for general trends $[223,417]$. The temperature dependence provides a simple way to tune the volume fraction in situ [111,352]. This feature has already been exploited in ageing experiments (see section 4.2).

One can also use sticky colloidal particles to study colloidal gelation. The most common way to induce gelation is to add a small polymer molecule to the colloidal suspension. A colloidal particle surrounded by small polymers feels an isotropic osmotic pressure whereas two particles close together feel an unbalanced osmotic pressure that pushes them together. The result is an effective attractive interaction between the particles, which has been termed the depletion force [135, 419]. The range of this interaction is set by the size of the small polymers (typically their radius of gyration is at least ten times smaller than the colloidal particle radius), and the strength of the interaction is set by the polymer concentration.

MCT made intriguing predictions of a re-entrant glass transition for hard spheres with a very short range depletion attraction [1,420-423], with supporting evidence from simulations [424]. A fascinating set of experiments confirmed these predictions [256-259, 407, 425, 426]. A state diagram is shown in figure 17: the filled symbols indicate glassy states. The re-entrant glass transition can be understood by considering the two types of glasses. At high polymer concentration, all of the colloidal particles stick together in a gel-an 'attractive glass' - indicated by the filled squares in figure 17. Within the gel, particles are at a high local volume fraction, whereas the pores of the gel are at a lower local volume fraction [427]. If the polymer concentration is decreased, at some point, particles detach from the gelled phase and rearrange, passing through the pores, and the sample becomes ergodic: that is, a fluid! These states are shown by the inverted triangles in figure 17, and eventually these samples all crystallized. If the polymer concentration is further decreased, the distinction between the gel and the pores is lost, and the system can become glassy again — a 'repulsive glass' indicated by the filled circles in figure 17. At this point, it is like the hard-sphere glass transition, where particle arrest is due to cages. Here the sample is much more isotropic, with the local volume fraction essentially constant and larger than $\phi_{\mathrm{g}}$. The intermediate fluid state allows for the possibility of high-volume fraction colloids with moderately low viscosities [407, 260].

For more about the physics of colloid-polymer mixtures, two classic articles are [428,429] and good reviews are [1, 430, 431]. Microscopy studies of particle motion comparing attractive and repulsive glasses include [288, 289]. Note that for very strong attractive forces and lower volume fractions, the sample is considered a colloidal gel rather than an attractive colloidal glass; the distinction is clarified in [408]. 


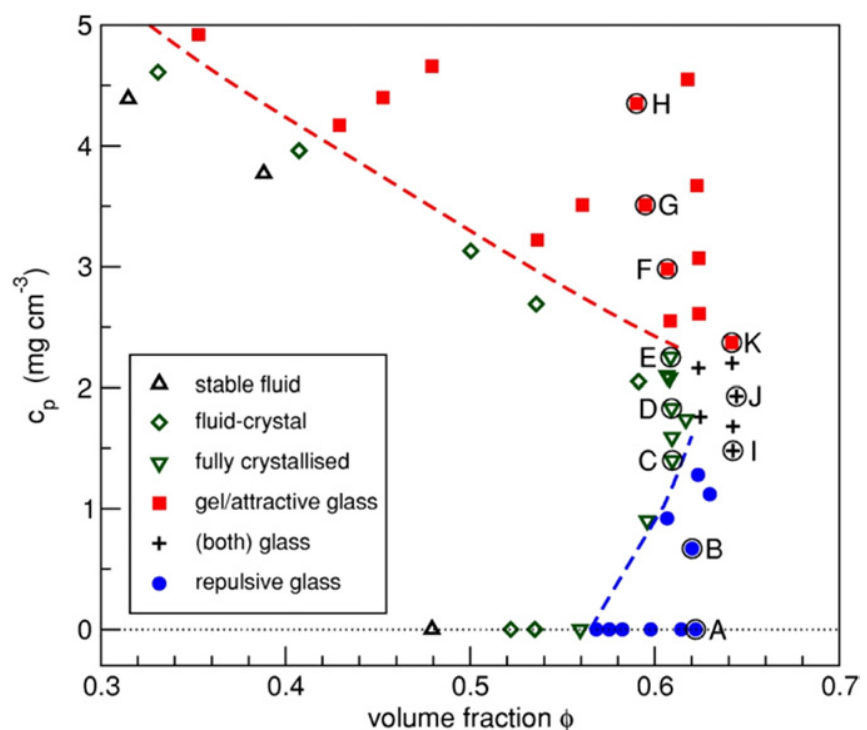

Figure 17. Phase diagram showing equilibrium and non-equilibrium states of a colloid-polymer mixture. Open symbols fully equilibrated, while filled symbols did not and thus correspond to the glassy states. The dashed curves are guides to the eye. $c_{\mathrm{p}}$ is the concentration of the added polymer, which had a size ratio (polymer radius of gyration to particle size) of $\xi=0.09$. (The letters indicate specific states discussed in [426].) Reprinted with permission from [426], copyright 2004 by the American Physical Society.

A more exotic colloid is laponite: nanometre-sized colloidal clay platelets. These aqueous suspensions have been observed to become viscous at very low solid concentrations [432,433] and exhibit a glass transition at remarkably low volume fractions of $\phi \approx 0.01-0.02$ [164, 434-436]. Along with rheology techniques, it is possible at such low concentrations to use DLS to study dynamics. One can also shear the suspension quickly to create highly reproducible initial liquid states. Given these advantages, laponite suspensions are commonly used in studies of ageing, in particular [343, 357, 372, 373, 375, 434, 435, 437, 438]. Many of these studies also relate to effective temperatures during ageing; see section 4.2 for more discussion of effective temperatures.

Emulsions are composed of droplets of one liquid immersed in a second immiscible liquid. The interfaces between the liquids are stabilized by surfactants; review articles discussing emulsions include [439,440]. The emulsion glass transition appears to be closer to random close-packing [441,442]. Emulsion glasses were recently used to study the forces between droplets in close-packed samples [443-445] and the structure of random close packing of polydisperse samples [446, 447].

Foams, bubbles of gas in a liquid, are yet another system that exhibits glassy behaviour [448, 449]. A common technique uses shear to study how the behaviour within the foam depends on shear rate. Shear in this case is used to "unjam" the foam, in contrast to increasing temperature to liquefy a glass or decreasing the volume fraction to unjam a colloidal glass. This method has been used to good effect [450-457]. One particularly thought-provoking simulation examined several different measures of an effective

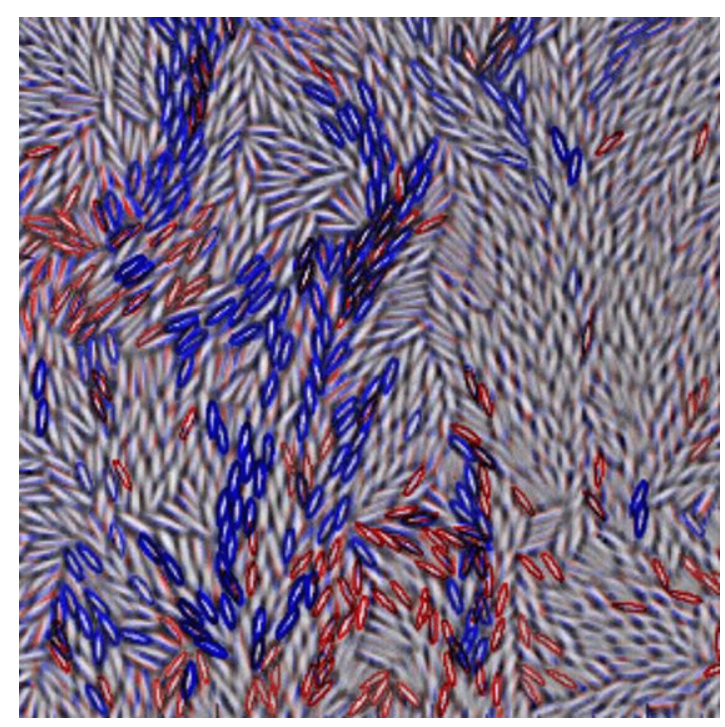

Figure 18. Ellipsoidal particles in a quasi-2D experiment. The ellipsoids that translate the most at this moment are outlined in blue (thick darker grey lines), and those that rotate the most are outlined in red (thin lighter grey lines). Those outlined in black translate and rotate. The ellipsoids are $3.33 \mu \mathrm{m}$ long. Reprinted with permission from [472], copyright 2011 by the American Physical Society (adapted from original image in [466]).

temperature of a sheared foam and found good agreement between the different measures [366]; see section 4.2 of this review for further discussion of effective temperatures.

Spin glasses, not yet mentioned in this review, are another large category of glass-forming systems [458]. There, glassy behaviour is controlled by range of interaction and the coupling strength between spins. It has been demonstrated that confocal microscopy observations of colloidal glasses can be mapped onto spin glass formalism [459].

\subsection{Future directions: anisotropic colloidal particles}

In recent years, a number of new methods have been successful in synthesizing non-spherical colloidal particles [460-463]. Several of these methods can produce particles suitable for colloidal glass studies; see, for example, [464, 465]. Anisotropic particles are potentially better able to mimic the molecular glass transition, which in many cases occurs with non-spherically symmetric molecules. A recent experiment studied the glass transition of colloidal ellipsoids in two dimensions [466] and found that rotational motions ceasedbecame glassy - at an area fraction lower than that for the translational motion. This confirmed theoretical predictions $[1,467]$. An image adapted from their experimental data is shown in figure 18, indicating that these particles rotate and translate in spatially dynamically heterogeneous ways (see section 3.3). Large translational motions typically occurred for particles within aligned domains, while the large rotation motions were more prevalent between the domains. This sort of experiment could easily test other predictions: for example, simulations and theory predict a non-monotonic dependence on aspect ratio [468-470]. The differing glass transitions for rotational and translational motions should disappear for aspect 
ratios closer to $1[467,471]$. Aspect ratios closer to 1 might be useful to study, given that molecular glasses only have a single glass transition [472].

Other possibilities including using 'patchy' colloids, which have attractive regions on their surfaces. Simulations suggest interesting gel phases can form [473,474]. A good review of experimental efforts to create patchy colloids and recent simulation results is [475].

A different take on breaking spherical symmetry is to use particles which are optically asymmetric, but spherical in other respects. Such particles have been created and their rotational motion can be tracked [476]. This is a fun technique, but probably needs to be combined with other techniques to give such particles some other non-spherical symmetry; otherwise, the spheres are unlikely to dramatically change their behaviour near the colloidal glass transition. While hydrodynamic interactions with nearby particles slow rotational motion, the regular colloidal glass transition, which is an inhibition of translational motion, would be unaffected.

The many other ways to make non-spherically symmetric particles are comprehensively reviewed in [477]. One could imagine, for example, tetrahedral particles with two sticky patches, perhaps with the sticky patches having some specified position on the tetrahedra. Studying the interplay of shape, interaction and the colloidal glass transition is likely to be fruitful for quite some time.

\section{Conclusion}

Colloidal suspensions are used in many ways to study the physics of the glass transition, as has been described throughout this review. As noted in the introduction, dense colloidal suspensions have material properties of potential interest and relevance to a variety of industrial processes, and so even without a connection to the glass transition, dense colloids are worthy of study. Aspects of the colloidal glass transition remain to be understood, such as the exact roles of gravity and polydispersity in suppressing crystallization, correlations between structure and dynamics, and the best way to define $\phi_{\mathrm{g}}$, among others. Given the rich physics in these systems and recent progress in synthesis techniques, the field is likely to continue flourishing for some time.

We consider a final pair of questions: how relevant are colloids as a model for the glass transition; and what is currently the 'best' value of $\phi_{\mathrm{g}}$ ? From the perspective of this review, the answer to the first question is clearly 'yes': the colloidal glass transition has numerous similarities to glass transitions in general and is therefore a good model system. Microscopic relaxation time scales in colloids grow dramatically (figures 2, 5 and 10), as does the macroscopic viscosity (figure 8). Colloidal glasses demonstrate an apparent variety of fragilities as shown in figure 11, although section 3.2 discusses the variety of interpretations that are possible for these data. Dense colloidal liquids exhibit spatially heterogeneous dynamics (figures 12 and 13) similar to molecular glasses, and the structure of colloidal glasses is amorphous (figure 14), similar to results from glassy simulations and molecular experiments. Colloidal glasses also exhibit ageing behaviour (figure 15) similar to molecular glasses, even matching in many details [352]. In general, results from colloids agree with glass transition theories qualitatively (figure 18) and often quantitatively (figures 5, 8 and 10).

The answer to our second question, concerning the 'best' value for $\phi_{\mathrm{g}}$, is one of two candidates: the traditional value $\phi_{\mathrm{g}} \approx 0.58$; and the value for random close packing, $\phi_{\mathrm{rcp}} \approx$ 0.64. Much of the data mentioned above has quantities diverging at 0.58 (figures 2, 5 and 10) or changing qualitatively at 0.58 (figures 13 and 15). There is one non-trivial exception: the viscosity data in figure 8 are well fit by a function diverging at 0.64 [221]. While Cheng et al were only able to measure a change in viscosity of four orders of magnitude, far less than is seen for molecular glasses, it is conceivable that the experimental $\phi_{\mathrm{g}} \approx 0.58$ is still below a truer glass transition point for colloids - perhaps at a volume fraction comparable to $\phi_{\text {rcp. }}$. The limitations of measuring larger viscosities are discussed in section 3.1.

However, volume fraction measurements are thought to have uncertainties of at least $\pm 3 \%$, which possibly lowers the divergence point of [221] from 0.64 to 0.62 ; the specific case of the data in [221] is discussed in [66]. A second possibility is simply to accept that the viscosity at $\phi_{\mathrm{g}}$, while 'large,' is still finite. The same is true for the viscosities of molecular glasses at $T_{\mathrm{g}}$, albeit at significantly larger values. Given the number of observations pointing to a glass transition at $\phi_{\mathrm{g}} \approx 0.58$ as well as complementary simulation results $[43,44]$, we feel that 0.58 is still a reasonable 'best' candidate for $\phi_{\mathrm{g}}$, although it is clear that there are important questions about this value that still remain (both for viscosity [221] and relaxation times [261,262]). It is certainly likely that the value $\phi_{\mathrm{g}} \approx 0.58$, and indeed the existence of a glass transition in general, hinges on having a polydisperse sample $[43,44]$ — which is easy to achieve in many experiments.

Over the volume fraction range for which samples can be equilibrated $(\phi \lesssim 0.6)$, colloids share many similarities with molecular glasses, both in experiments and simulations (as described above). These similarities strengthen the utility of the colloidal model system, despite the relatively limited viscosity range that is observable. Simulations are also somewhat limited in the time scales that they can address [219]. As noted in section 2.6, the agreement between colloidal experiments and computational simulations strengthens the validity of both. At this point in time, 26 years after the first experimental demonstration of the colloidal hard-sphere glass transition by Pusey and van Megen [30], many points of similarity between the colloidal glass transition at $\phi_{\mathrm{g}}$ and the molecular glass transition have been found and studied. Thus, to answer our first question once more: colloidal glasses are indeed a useful model for understanding the glass transition.

Throughout this review, we have pointed out earlier sources where topics are discussed in more detail, and so we conclude by noting prior reviews of the colloidal glass transition, of which several have already been referenced here [1, 49, 72, 431, 478]. 


\section{Acknowledgments}

We thank R Besseling, L Berthier, P M Chaikin, A J Liu, D Reichman and C B Roth for helpful discussions. This work was supported by a grant from the National Science Foundation (CHE-0910707).

\section{References}

[1] Sciortino F and Tartaglia P 2005 Adv. Phys. 54 471-524

[2] Götze W and Sjögren L 1992 Rep. Prog. Phys. 55 241-376

[3] Angell C A 1995 Science 267 1924-35

[4] Stillinger F H 1995 Science 267 1935-9

[5] Ediger M D, Angell C A and Nagel S R 1996 J. Phys. Chem. $10013200-12$

[6] Angell C A 2000 J. Phys.: Condens. Matter 12 6463-75

[7] Debenedetti P G and Stillinger F H 2001 Nature 410 259-67

[8] Andersen H C 2005 Proc. Natl Acad. Sci. USA 102 6686-91

[9] Dyre J C 2006 Rev. Mod. Phys. 78 953-72

[10] Berthier L and Biroli G 2011 Rev. Mod. Phys. 83 587-645

[11] Roland C, Hensel-Bielowka S, Paluch M and Casalini R 2005 Rep. Prog. Phys. 68 1405-78

[12] Win K Z and Menon N 2006 Phys. Rev. E 73040501

[13] Lindsay H M and Chaikin P M 1982 J. Chem. Phys. $763774-81$

[14] Zanotto E D 1998 Am. J. Phys. 66 392-5

[15] Zanotto E D and Gupta P K 1999 Am. J. Phys. 67 260-2

[16] Edgeworth R, Dalton B J and Parnell T 1984 Euro. J. Phys. 5 198-200

[17] Vincent B 2005 Introduction to Colloidal Dispersions (Oxford: Blackwell) chapter 1

[18] Larson R G 1998 The Structure and Rheology of Complex Fluids (Oxford: Oxford University Press)

[19] Hiltner P A and Krieger I M 1969 J. Phys. Chem. 73 2386-9

[20] Hiltner P A, Papir Y S and Krieger I M 1971 J. Phys. Chem. 75 1881-6

[21] Kose A, Ozaki M, Takano K, Kobayashi Y and Hachisu S 1973 J. Colloid Interface Sci. 44 330-8

[22] Hastings R 1978 J. Chem. Phys. 68 675-8

[23] Aastuen D J W, Clark N A, Cotter L K and Ackerson B J 1986 Phys. Rev. Lett. 57 1733-6

[24] Van Winkle D H and Murray C A 1986 Phys. Rev. A $34562-73$

[25] Murray C A and Van Winkle D H 1987 Phys. Rev. Lett. 58 1200-3

[26] Sirota E B, Yang H D O, Sinha S K, Chaikin P M, Axe J D and Fujii Y 1989 Phys. Rev. Lett. 62 1524-7

[27] Murray C A, Sprenger W O and Wenk R A 1990 Phys. Rev. B 42 688-703

[28] Marshall L and Zukoski C F 1990 J. Phys. Chem. 94 1164-71

[29] Murray C A and Grier D G 1996 Annu. Rev. Phys. Chem. 47 421-62

[30] Pusey P N and van Megen W 1986 Nature 320 340-2

[31] Rosenberg R O, Thirumalai D and Mountain R D 1989 J. Phys.: Condens. Matter 1 2109-14

[32] Pusey P N and van Megen W 1987 Phys. Rev. Lett. 59 2083-6

[33] Pusey P N 1987 J. Physique 48 709-12

[34] Bernal J D 1964 Proc. R. Soc. Lond. A 280 299-322

[35] Löwen H 2000 Fun with Hard Spheres vol 554 (Berlin: Springer) pp 295-331

[36] Donev A, Stillinger F H and Torquato S 2007 J. Chem. Phys. 127124509

[37] Wood W W and Jacobson J D 1957 J. Chem. Phys. 27 1207-8

[38] Alder B J and Wainwright T E 1957 J. Chem. Phys. 27 1208-9

[39] Hoover W G and Ree F H 1967 J. Chem. Phys. 47 4873-8
[40] Bolhuis P G and Kofke D A 1996 Phys. Rev. E 54 634-43

[41] Auer S and Frenkel D 2001 Nature 413 711-13

[42] Schöpe H J, Bryant G and van Megen W 2007 J. Chem. Phys. 127084505

[43] Zaccarelli E, Valeriani C, Sanz E, Poon W C K, Cates M E and Pusey P N 2009 Phys. Rev. Lett. 103135704

[44] Pusey P N, Zaccarelli E, Valeriani C, Sanz E, Poon W C K and Cates M E 2009 Phil. Trans. R. Soc. Lond. A 367 4993-5011

[45] Fasolo M and Sollich P 2003 Phys. Rev. Lett. 91068301

[46] Sollich P and Wilding N B 2010 Phys. Rev. Lett. 104118302

[47] Hynninen A P and Dijkstra M 2003 Phys. Rev. E 68021407

[48] Hernández-Guzmán J and Weeks E R 2009 Proc. Natl Acad. Sci. USA 106 15198-202

[49] Härtl W 2001 Curr. Opin. Colloid Interface Sci. 6 479-83

[50] Bernal J D and Mason J 1960 Nature 188 910-11

[51] Torquato S, Truskett T M and Debenedetti P G 2000 Phys. Rev. Lett. 84 2064-7

[52] Radin C 2008 J. Stat. Phys. 131 567-73

[53] Hermes M and Dijkstra M 2010 Europhys. Lett. 8938005

[54] Snook I, van Megen W and Pusey P 1991 Phys. Rev. A 43 6900-7

[55] Speedy R J 1998 Mol. Phys. 95 169-78

[56] Doliwa B and Heuer A 1998 Phys. Rev. Lett. 80 4915-18

[57] Németh Z T and Löwen H 1999 Phys. Rev. E 59 6824-9

[58] Foffi G, Götze W, Sciortino F, Tartaglia P and Voigtmann T 2004 Phys. Rev. E 69011505

[59] Xu N, Haxton T K, Liu A J and Nagel S R 2009 Phys. Rev. Lett. 103245701

[60] Bryant G, Williams S R, Qian L, Snook I K, Perez E and Pincet F 2002 Phys. Rev. E 66060501

[61] Underwood S M, Taylor J R and van Megen W 1994 Langmuir 10 3550-4

[62] Antl L, Goodwin J W, Hill R D, Ottewill R H, Owens S M Papworth S and Waters J A 1986 Colloids Surf. 17 67-78

[63] Bosma G, Pathmamanoharana C, de Hooga E H A, Kegel W K, van Blaaderen A and Lekkerkerker H N W 2002 J. Colloid Interface Sci. 245 292-300

[64] Campbell A I and Bartlett P 2002 J. Colloid Interface Sci. $256325-30$

[65] Elsesser M T and Hollingsworth A D 2010 Langmuir 26 17989-96

[66] Poon W C K, Weeks E R and Royall C P 2012 Soft Matter $821-30$

[67] Dinsmore A D, Weeks E R, Prasad V, Levitt A C and Weitz D A 2001 App. Opt. 40 4152-9

[68] Simeonova N B and Kegel W K 2004 Phys. Rev. Lett. 93035701

[69] Zhu J, Li M, Rogers R, Meyer W, Ottewill R H, STS-73 Space Shuttle Crew, Russel W B and Chaikin P M 1997 Nature 387 883-5

[70] Kegel W K 2000 Langmuir 16 939-41

[71] Bartsch E 1998 Curr. Opin. Colloid Interface Sci. 3 577-85

[72] Schweizer K S and Yatsenko G 2007 J. Chem. Phys. 127164505

[73] Di Cola E, Moussaïd A, Sztucki M, Narayanan T and Zaccarelli E 2009 J. Chem. Phys. 131144903

[74] Gleim T, Kob W and Binder K 1998 Phys. Rev. Lett. 81 4404-7

[75] Szamel G and Flenner E 2004 Europhys. Lett. 67 779-85

[76] Tokuyama M, Yamazaki H and Terada Y 2003 Phys. Rev. E 67062403

[77] Tokuyama M 2007 Physica A 378 157-66

[78] Berthier L, Biroli G, Bouchaud J P, Kob W, Miyazaki K and Reichman D R 2007 J. Chem. Phys. 126184503

[79] Höfling F, Munk T, Frey E and Franosch T 2008 J. Chem. Phys. 128164517

[80] Puertas A M 2010 J. Phys.: Condens. Matter 22104121 
[81] Henderson S I, Mortensen T C, Underwood S M and van Megen W 1996 Physica A 233 102-16

[82] Henderson S I and van Megen W 1998 Phys. Rev. Lett. 80 877-80

[83] Kob W and Andersen H C 1995 Phys. Rev. E 51 4626-41

[84] Kob W and Andersen H C 1995 Phys. Rev. E 52 4134-53

[85] Meller A and Stavans J 1992 Phys. Rev. Lett. 68 3646-9

[86] Sutherland W 1905 Phil. Mag. 9 781-5

[87] Einstein A 1905 Ann. Phys., Lpz. 17 549-60

[88] Pusey P N and Tough R J A 1983 Faraday Discuss. Chem. Soc. 76 123-36

[89] Snook I, van Megen W and Tough R J A 1983 J. Chem. Phys. $785825-36$

[90] Beenakker C W J and Mazur P 1983 Phys. Lett. A 98 22-4

[91] Beenakker C W J and Mazur P 1984 Physica A 126 349-70

[92] van Megen W, Ottewill R H, Owens S M and Pusey P N 1985 J. Chem. Phys. 82 508-15

[93] Berne B J, Boon J P and Rice S A 1966 J. Chem. Phys. 45 1086-96

[94] Sjögren L 1980 Phys. Rev. A 22 2883-90

[95] Wahnstrom G and Sjögren L 1982 J. Phys. C: Solid State Phys. 15401

[96] Rabani E, Gezelter J D and Berne B J 1997 J. Chem. Phys. $1076867-76$

[97] Verberg R, Schepper I M D and Cohen E G D 1999 Europhys. Lett. 48 397-402

[98] Schweizer K S and Saltzman E J 2003 J. Chem. Phys. 119 1181-96

[99] Weeks E R and Weitz D A 2002 Chem. Phys. 284 361-7

[100] van Megen W, Mortensen T C, Williams S R and Müller J 1998 Phys. Rev. E 58 6073-85

[101] Batchelor G K 1972 J. Fluid Mech. 52 245-68

[102] Paulin S E and Ackerson B J 1990 Phys. Rev. Lett. 64 2663-6

[103] He P, Mejia A F, Cheng Z, Sun D, Sue H J, Dinair D S and Marquez M 2010 Phys. Rev. E 81026310

[104] Bartsch E, Antonietti M, Schupp W and Sillescu H 1992 J. Chem. Phys. 97 3950-63

[105] Bartsch E, Frenz V, Baschnagel J, Schärtl W and Sillescu H 1997 J. Chem. Phys. 106 3743-56

[106] Cavagna A 2009 Phys. Rep. 476 51-124

[107] Haw M D 2002 J. Phys.: Condens. Matter 14 7769-79

[108] Olafsen J (ed) 2010 Experimental and Computational Techniques in Soft Condensed Matter Physics 1st edn (Cambridge: Cambridge University Press)

[109] Elliot M and Poon W C K 2001 Adv. Colloid Interface Sci. 92 133-194

[110] Marcus A H, Schofield J and Rice S A 1999 Phys. Rev. E 60 5725-36

[111] Yunker P, Zhang Z, Aptowicz K and Yodh A G 2009 Phys. Rev. Lett. 10311570

[112] König H, Hund R, Zahn K and Maret G 2005 Eur. Phys. J. E 18 287-93

[113] Ebert F, Dillmann P, Maret G and Keim P 2009 Rev. Sci. Instrum. 80083902

[114] Royall C P, Leunissen M E and Blaaderen A V 2003 J. Phys.: Condens. Matter 15 S3581-96

[115] Yethiraj A and van Blaaderen A 2003 Nature 421 513-17

[116] Yethiraj A 2007 Soft Matter 3 1099-115

[117] Inoué S and Spring K R 1997 Video Microscopy : The Fundamentals (The Language of Science) 2nd edn (Berlin: Springer)

[118] Habdas P and Weeks E R 2002 Curr. Opin. Colloid Interface Sci. 7 196-203

[119] Prasad V, Semwogerere D and Weeks E R 2007 J. Phys.: Condens. Matter 19113102

[120] Draaijer A and Houpt P M 1988 Scanning 10 139-45

[121] Awamura D, Ode T and Yonezawa M 1990 Science on Form: 3D Dynamic Morphometry for Bridge between Structure and Function, Proc. 2nd Int. Symp. for Science on Form ed S Ishizaka (Tokyo: KTK Scientific Publishers) pp 81-95

[122] Xiao G Q, Corle T R and Kino G S 1988 Appl. Phys. Lett. $53716-18$

[123] Yoshida H, Ito K and Ise N 1991 Phys. Rev. B 44 435-8

[124] van Blaaderen A, Imhof A, Hage W and Vrij A 1992 Langmuir 8 1514-7

[125] Ito K, Yoshida H and Ise N 1994 Science 263 66-8

[126] van Blaaderen A 1993 Adv. Mater. 5 52-4

[127] van Blaaderen A and Wiltzius P 1995 Science 270 1177-9

[128] Kegel W K and van Blaaderen A 2000 Science 287 290-3

[129] Weeks E R, Crocker J C, Levitt A C, Schofield A and Weitz D A 2000 Science 287 627-31

[130] Chestnut M H 1997 Curr. Opin. Colloid Interface Sci. 2 158-61

[131] Pawley J 2006 Handbook of Biological Confocal Microscopy 3rd edn (Berlin: Springer)

[132] Crocker J C and Grier D G 1996 J. Colloid Interface Sci. 179 298-310

[133] Gao Y and Kilfoil M L 2009 Opt. Express 17 4685-704

[134] Jenkins M C and Egelhaaf S U 2008 Adv. Colloid Interface Sci. 136 65-92

[135] Crocker J C, Matteo J A, Dinsmore A D and Yodh A G 1999 Phys. Rev. Lett. 82 4352-5

[136] Baumgartl J and Bechinger C 2005 Europhys. Lett. 71 487-93

[137] Weeks E R and Weitz D A 2002 Phys. Rev. Lett. 89095704

[138] Weeks E R and Crocker J C 2012 Particle tracking using IDL http://www.physics.emory.edu/ weeks/idl/

[139] Besseling R, Isa L, Weeks E R and Poon W C K 2009 Adv Colloid Interface Sci. 146 1-17

[140] Berne B J and Pecora R 1976 Dynamic Light Scattering (New York: Wiley)

[141] Jones R B and Pusey P N 1991 Annu. Rev. Phys. Chem. 42 137-69

[142] van Megen W, Underwood S M and Snook I 1986 J. Chem. Phys. 85 4065-72

[143] Williams S R and van Megen W 2001 Phys. Rev. E 64041502

[144] Hoffman R L 1992 J. Rheol. 36 947-65

[145] D'Haene P and Mewis J 1994 Rheol. Acta 33 165-74

[146] Lynch J M, Cianci G C and Weeks E R 2008 Phys. Rev. E 78031410

[147] Götze W and Voigtmann T 2003 Phys. Rev. E 67021502

[148] Foffi G, Götze W, Sciortino F, Tartaglia P and Voigtmann T 2003 Phys. Rev. Lett. 91085701

[149] Zaccarelli E, Löwen H, Wessels P P F, Sciortino F, Tartaglia P and Likos C N 2004 Phys. Rev. Lett. 92225703

[150] Pusey P N and van Megen W 1989 Physica A 157 705-41

[151] Cipelletti L and Ramos L 2002 Curr. Opin. Colloid Interface Sci. 7 228-34

[152] Scheffold F and Cerbino R 2007 Curr. Opin. Colloid Interface Sci. 12 50-7

[153] Pusey P 1999 Curr. Opin. Colloid Interface Sci. 4 177-85

[154] Maret G and Wolf P E 1987 Z. Phys. B 65 409-13

[155] Wolf P E, Maret G, Akkermans E and Maynard R 1988 J. Physique 49 63-75

[156] Akkermans E, Wolf P E, Maynard R and Maret G 1988 J. Physique 49 77-98

[157] Pine D J, Weitz D A, Chaikin P M and Herbolzheimer E 1988 Phys. Rev. Lett. $601134-7$

[158] Pine D J, Weitz D A, Zhu J X and Herbolzheimer E 1990 J. Physique 51 2101-27

[159] Maret G 1997 Curr. Opin. Colloid Interface Sci. 2 251-7

[160] Klein R and Nägele G 1996 Curr. Opin. Colloid Interface Sci. 1 4-10

[161] Bhatia S 2005 Curr. Opin. Colloid Interface Sci. 9 404-11

[162] Berthier L, Biroli G, Bouchaud J P, Cipelletti L and van Saarloos W 2011 Dynamical Heterogeneities in Glasses, Colloids, and Granular Media (International Series of 
Monographs on Physics) (Oxford: Oxford University Press)

[163] Mason T G and Weitz D A 1995 Phys. Rev. Lett. 75 2770-3

[164] Mohan P H and Joshi Y M 2010 Soft Matter 6 1462-6

[165] Puertas A M, Zaccarelli E and Sciortino F 2005 J. Phys.: Condens. Matter 17 L271-L277

[166] Pham K N, Petekidis G, Vlassopoulos D, Egelhaaf S U, Pusey P N and Poon W C K 2006 Europhys. Lett. 75 624-30

[167] Conrad J C, Dhillon P P, Weeks E R, Reichman D R and Weitz D A 2006 Phys. Rev. Lett. 97265701

[168] de Schepper I M, Smorenburg H E and Cohen E G D 1993 Phys. Rev. Lett. 70 2178-81

[169] Mason T G and Weitz D A 1995 Phys. Rev. Lett. 74 1250-3

[170] Mason T G, Ganesan K, van Zanten J H, Wirtz D and Kuo S C 1997 Phys. Rev. Lett. 79 3282-5

[171] Crocker J C, Valentine M T, Weeks E R, Gisler T, Kaplan P D, Yodh A G and Weitz D A 2000 Phys. Rev. Lett. 85 888-91

[172] Breedveld V and Pine D J 2003 J. Mater. Sci. 38 4461-70

[173] Waigh T A 2005 Rep. Prog. Phys. 68 685-742

[174] Habdas P, Schaar D, Levitt A C and Weeks E R 2004 Europhys. Lett. 67 477-83

[175] Squires T M and Brady J F 2005 Phys. Fluids 17073101

[176] Carpen I C and Brady J F 2005 J. Rheol. 49 1483-502

[177] Drocco J A, Hastings M B, Reichhardt C J O and Reichhardt C 2005 Phys. Rev. Lett. 95088001

[178] Williams S R and Evans D J 2006 Phys. Rev. Lett. 96015701

[179] Meyer A, Marshall A, Bush B G and Furst E M 2006 J. Rheol. 50 77-92

[180] Reichhardt C and Reichhardt C J O 2006 Phys. Rev. Lett. 96028301

[181] Zangi R, Mackowiak S A and Kaufman L J 2007 J. Chem. Phys. 126104501

[182] Gazuz I, Puertas A M, Voigtmann T and Fuchs M 2009 Phys. Rev. Lett. 102248302

[183] Khair A S and Squires T M 2010 Phys. Rev. Lett. 105156001

[184] Wilson L G, Harrison A W, Poon W C K and Puertas A M 2011 Europhys. Lett. 9358007

[185] Bergenholtz J 2001 Curr. Opin. Colloid Interface Sci. 6 484-8

[186] Lionberger R A and Russel W B 2000 Adv. Chem. Phys. 111 399-474

[187] Stickel J J and Powell R L 2005 Annu. Rev. Fluid Mech. 37 129-49

[188] Buscall R 2010 J. Rheol. 54 1177-83

[189] Brader J M 2010 J. Phys.: Condens. Matter 22363101

[190] Macosko C W 1994 Rheology: Principles, Measurements, and Applications (Advances in Interfacial Engineering) 1st edn (New York: Wiley)

[191] Jones R A L 2002 Soft Condensed Matter (Oxford Master Series in Condensed Matter Physics vol 6) 1st edn (Oxford: Oxford University Press)

[192] Chen D T N, Wen Q, Janmey P A, Crocker J C and Yodh A G 2010 Annu. Rev. Condens. Matter Phys. 1 301-22

[193] Brady J F and Bossis G 1988 Annu. Rev. Fluid Mech. 20 111-57

[194] Kob W, Donati C, Plimpton S J, Poole P H and Glotzer S C 1997 Phys. Rev. Lett. 79 2827-30

[195] Poole P H, Donati C and Glotzer S C 1998 Physica A 261 51-9

[196] Donati C, Glotzer S C and Poole P H 1999 Phys. Rev. Lett. 82 5064-7

[197] Berthier L 2004 Phys. Rev. E $69020201(\mathrm{R})$

[198] Doliwa B and Heuer A 2000 Phys. Rev. E 61 6898-908

[199] Hurley M M and Harrowell P 1995 Phys. Rev. E 52 1694-8
[200] Hurley M M and Harrowell P 1996 J. Chem. Phys. 105 10521-6

[201] Yamamoto R and Onuki A 1998 Phys. Rev. E 58 3515-29

[202] Kurita R and Weeks E R 2010 Phys. Rev. E 82041402

[203] Widmer-Cooper A, Harrowell P and Fynewever H 2004 Phys. Rev. Lett. 93135701

[204] Donati C, Douglas J F, Kob W, Plimpton S J, Poole P H and Glotzer S C 1998 Phys. Rev. Lett. 80 2338-41

[205] Kasper A, Bartsch E and Sillescu H 1998 Langmuir 14 5004-10

[206] van Meel J A, Frenkel D and Charbonneau P 2009 Phys. Rev. E 79 030201(R)

[207] van Meel J A, Charbonneau B, Fortini A and Charbonneau P 2009 Phys. Rev. E 80061110

[208] Charbonneau P, Ikeda A, van Meel J A and Miyazaki K 2010 Phys. Rev. E 81 040501(R)

[209] Kob W, Scheidler P and Binder K 2000 Europhys. Lett. 52 277-83

[210] Kim K and Yamamoto R 2000 Phys. Rev. E 61 R41-4

[211] Murray C 1998 MRS Bull. 23 33-8

[212] Archer A J, Hopkins P and Schmidt M 2007 Phys. Rev. E $\mathbf{7 5} 040501$

[213] Nugent C R, Edmond K V, Patel H N and Weeks E R 2007 Phys. Rev. Lett. 99025702

[214] Desmond K W and Weeks E R 2009 Phys. Rev. E 80051305

[215] Edmond K V, Nugent C R and Weeks E R 2010 Eur. Phys. J. Spec. Top. 189 83-93

[216] Widmer-Cooper A and Harrowell P 2005 J. Phys.: Condens. Matter 17 S4025-34

[217] Santen L and Krauth W 2000 Nature 405 550-1

[218] Frenkel D and Smit B 2001 Understanding Molecular Simulation, From Algorithms to Applications (Computational Science) 2nd edn (New York: Academic)

[219] Glotzer S C 2000 J. Non-Cryst. Solids 274 342-55

[220] Habdas P, Weeks E R and Lynn D G 2006 Phys. Teach. $44276-9$

[221] Cheng Z, Zhu J, Chaikin P M, Phan S E and Russel W B 2002 Phys. Rev. E 65041405

[222] Crassous J J, Siebenbürger M, Ballauff M, Drechsler M, Hajnal D, Henrich O and Fuchs M 2008 J. Chem. Phys. 128204902

[223] Siebenbürger M, Fuchs M, Winter H and Ballauff M 2009 J. Rheol. 53 707-26

[224] Yamamoto R and Onuki A 1997 Europhys. Lett. 40 61-6

[225] Phan S E, Russel W B, Cheng Z, Zhu J, Chaikin P M, Dunsmuir J H and Ottewill R H 1996 Phys. Rev. E 54 6633-45

[226] Pusey P, Segre P, Behrend O, Meeker S and Poon W 1997 Physica A 235 1-8

[227] Mewis J, Frith W J, Strivens T A and Russel W B 1989 AIChE J. 35 415-22

[228] Doolittle A K 1951 J. App. Phys. 22 1471-5

[229] Tarjus G and Kivelson D 2001 The Viscous Slowing Down of Supercooled Liquids and the Glass Transition: Phenomonology, Concepts, and Models 1st edn (London: Taylor and Francis) pp 20-38

[230] Woodcock L V and Angell C A 1981 Phys. Rev. Lett. 47 1129-32

[231] Hecksher T, Nielsen A I, Olsen N B and Dyre J C 2008 Nature Phys. 4 737-41

[232] McKenna G B 2008 Nature Phys. 4673

[233] Olsson P and Teitel S 2007 Phys. Rev. Lett. 99178001

[234] Olsson P and Teitel S 2011 Phys. Rev. E 83030302

[235] Vaagberg D, Balderas D V, Moore M A, Olsson P and Teitel S 2011 Phys. Rev. E 83030303

[236] Ediger M D 2000 Annu. Rev. Phys. Chem. 51 99-128

[237] Ngai K L and Rendell R W 1998 Phil. Mag. B 77 621-31

[238] Ngai K 2007 J. Non-Cryst. Solids 353 709-18 
[239] Segrè P N, Meeker S P, Pusey P N and Poon W C K 1995 Phys. Rev. Lett. 75 958-61

[240] Fujara F, Geil B, Sillescu H and Fleischer G 1992 Z. Phys. B 88 195-204

[241] Cicerone M T and Ediger M D 1993 J. Phys. Chem. 97 10489-97

[242] Chang I, Fujara F, Geil B, Heuberger G, Mangel T and Sillescu H 1994 J. Non-Cryst. Solids 172-174 248-55

[243] de Schepper I M, Cohen E G D and Verberg R 1996 Phys. Rev. Lett. 77584

[244] Segrè P N, Meeker S P, Pusey P N and Poon W C K 1996 Phys. Rev. Lett. 77585

[245] Bonn D and Kegel W K 2003 J. Chem. Phys. 118 2005-9

[246] Imhof A, van Blaaderen A, Maret G, Mellema J and Dhont J K G 1994 J. Chem. Phys. 100 2170-81

[247] van Megen W and Underwood S M 1994 Phys. Rev. E 49 4206-20

[248] van Megen W and Underwood S M 1988 J. Chem. Phys. 88 7841-6

[249] van Megen W and Underwood S M 1989 J. Chem. Phys. 91 552-9

[250] van Megen W and Pusey P N 1991 Phys. Rev. A 43 5429-41

[251] van Megen W, Martinez V A and Bryant G 2009 Phys. Rev. Lett. 102168301

[252] Götze W and Sjögren L 1991 Phys. Rev. A 43 5442-8

[253] Weysser F, Puertas A M, Fuchs M and Voigtmann T 2010 Phys. Rev. E 82011504

[254] Bartsch E, Frenz V, Moller S and Sillescu H 1993 Physica A $201363-71$

[255] Bartsch E 1995 J. Non-Cryst. Solids 192/193 384-92

[256] Bartsch E, Eckert T, Pies C and Sillescu H 2002 J. Non-Cryst. Solids 307-310 802-11

[257] Eckert T and Bartsch E 2002 Phys. Rev. Lett. 89125701

[258] Eckert T and Bartsch E 2003 Faraday Discuss. 123 51-64

[259] Eckert T and Bartsch E 2004 J. Phys.: Condens. Matter 16 S4937-S4950

[260] Willenbacher N, Vesaratchanon J S, Thorwarth O and Bartsch E 2011 Soft Matter 7 5777-88

[261] Brambilla G, El Masri D E M, Pierno M, Berthier L, Cipelletti L, Petekidis G and Schofield A B 2009 Phys. Rev. Lett. 102085703

[262] El Masri D, Brambilla G, Pierno M, Petekidis G, Schofield A B, Berthier L and Cipelletti L 2009 J. Stat. Mech. 2009 P07015

[263] van Megen W and Williams S R 2010 Phys. Rev. Lett. 104169601

[264] Brambilla G, Masri D E, Pierno M, Berthier L, Cipelletti L, Petekidis G and Schofield A 2010 Phys. Rev. Lett. 104169602

[265] Reinhardt J, Weysser F and Fuchs M 2010 Phys. Rev. Lett. 105199604

[266] Brambilla G, Masri D E, Pierno M, Berthier L and Cipelletti L 2010 Phys. Rev. Lett. 105199605

[267] Tokuyama M and Oppenheim I 1994 Phys. Rev. E 50 R16-R19

[268] Tokuyama M and Oppenheim I 1995 Physica A 216 85-119

[269] van Megen W, Martinez V A and Bryant G 2009 Phys. Rev. Lett. 103258302

[270] Reichman D R and Charbonneau P 2005 J. Stat. Mech. 2005 P05013

[271] Zaccarelli E, Foffi G, Gregorio P D, Sciortino F, Tartaglia P and Dawson K A 2002 J. Phys.: Condens. Matter $142413-37$

[272] Berthier L and Witten T A 2009 Phys. Rev. E 80021502

[273] Hodge I 1996 J. Non-Cryst. Solids 202 164-72

[274] Martinez L M and Angell C A 2001 Nature 410 663-7
[275] Huang D, Colucci D M and McKenna G B 2002 J. Chem. Phys. 116 3925-34

[276] Mattsson J, Wyss H M, Fernandez-Nieves A, Miyazaki K, Hu Z, Reichman D R and Weitz D A 2009 Nature $46283-6$

[277] Michele C D, Sciortino F and Coniglio A 2004 J. Phys.: Condens. Matter 16 L489-94

[278] Berthier L, Flenner E, Jacquin H and Szamel G 2010 Phys. Rev. E 81031505

[279] Barker J A and Henderson D 1976 Rev. Mod. Phys. 48 587-671

[280] Berthier L and Witten T A 2009 Europhys. Lett. 8610001

[281] Stieger M, Pedersen J S, Lindner P and Richtering W 2004 Langmuir 20 7283-92

[282] Eckert T and Richtering W 2008 J. Chem. Phys. 129124902

[283] Senff H and Richtering W 1999 J. Chem. Phys. 111 1705-11

[284] Sillescu H 1999 J. Non-Cryst. Solids 243 81-108

[285] Richert R 2002 J. Phys.: Condens. Matter 14 R703-R738

[286] Adam G and Gibbs J H 1965 J. Chem. Phys. 43 139-46

[287] Mazoyer S, Ebert F, Maret G and Keim P 2009 Europhys. Lett. 8866004

[288] Kaufman L J and Weitz D A 2006 J. Chem. Phys. 125074716

[289] Latka A, Han Y, Alsayed A M, Schofield A B, Yodh A G and Habdas P 2009 Europhys. Lett. 8658001

[290] Weeks E R, Crocker J C and Weitz D A 2007 J. Phys.: Condens. Matter 19205131

[291] Courtland R E and Weeks E R 2003 J. Phys.: Condens. Matter 15 S359-S365

[292] Donati C, Glotzer S C, Poole P H, Kob W and Plimpton S J 1999 Phys. Rev. E 60 3107-19

[293] Karmakar S, Dasgupta C and Sastry S 2009 Proc. Natl Acad. Sci. USA 1063675

[294] Glotzer S C, Novikov V N and Schrøder T B 2000 J. Chem. Phys. 112 509-12

[295] Lačević N, Starr F W, Schrøder T B and Glotzer S C 2003 J. Chem. Phys. $1197372-87$

[296] Conrad J C, Starr F W and Weitz D A 2005 J. Phys. Chem. B $10921235-40$

[297] Kurita R and Weeks E R 2011 arXiv:1112.1460

[298] Matharoo G S, Razul M S G and Poole P H 2006 Phys. Rev. E 74050502

[299] Berthier L and Jack R L 2007 Phys. Rev. E 76041509

[300] Cipelletti L, Bissig H, Trappe V, Ballesta P and Mazoyer S 2003 J. Phys.: Condens. Matter 15 S257-62

[301] Berthier L, Chandler D and Garrahan J P 2005 Europhys. Lett. $69320-6$

[302] Keys A S, Abate A R, Glotzer S C and Durian D J 2007 Nature Phys. 3 260-4

[303] Berthier L, Biroli G, Bouchaud J P, Kob W, Miyazaki K and Reichman D R 2007 J. Chem. Phys. 126184504

[304] Ballesta P, Duri A and Cipelletti L 2008 Nature Phys. 4 550-4

[305] Sarangapani P, Zhao J and Zhu Y 2008 J. Chem. Phys. 129104514

[306] Narumi T, Franklin S V, Desmond K W, Tokuyama M and Weeks E R 2011 Soft Matter 7 1472-82

[307] Alba-Simionesco C, Coasne B, Dosseh G, Dudziak G, Gubbins K E, Radhakrishnan R and Sliwinska-Bartkowiak M 2006 J. Phys.: Condens. Matter 18 R15-R68

[308] Alcoutlabi M and McKenna G B 2005 J. Phys.: Condens. Matter 17 R461-R524

[309] Roth C B and Dutcher J R 2005 J. Electroanal. Chem. $58413-22$

[310] Jackson C L and McKenna G B 1991 J. Non-Cryst. Solids 131-133 221-4

[311] Barut G, Pissis P, Pelster R and Nimtz G 1998 Phys. Rev Lett. $803543-6$

[312] Schüller J, Mel'nichenko Y, Richert R and Fischer E W 1994 Phys. Rev. Lett. 73 2224-7 
[313] Morineau D, Xia Y and Simionesco C A 2002 J. Chem. Phys. 117 8966-72

[314] Ngai K L 2002 Phil. Mag. B 82 291-303

[315] Ellison C J and Torkelson J M 2003 Nature Mater. 2 695-700

[316] Priestley R D, Ellison C J, Broadbelt L J and Torkelson J M 2005 Science 309 456-9

[317] Roth C B, McNerny K L, Jager W F and Torkelson J M 2007 Macromolecules 40 2568-74

[318] Richert R 2011 Annu. Rev. Phys. Chem. 62 65-84

[319] Bureau L 2010 Phys. Rev. Lett. 104218302

[320] Thompson P A, Grest G S and Robbins M O 1992 Phys. Rev. Lett. 68 3448-51

[321] Sarangapani P S and Zhu Y 2008 Phys. Rev. E 77010501

[322] Goel G, Krekelberg W P, Errington J R and Truskett T M 2008 Phys. Rev. Lett. 100106001

[323] Mittal J, Truskett T M, Errington J R and Hummer G 2008 Phys. Rev. Lett. 100145901

[324] Kurita R and Weeks E R 2010 Phys. Rev. E 82011403

[325] Donev A, Stillinger F H and Torquato S 2005 Phys. Rev. Lett. 95090604

[326] Torquato S and Stillinger F H 2003 Phys. Rev. E 68041113

[327] Zachary C E, Jiao Y and Torquato S 2011 Phys. Rev. Lett. 106178001

[328] Zachary C E, Jiao Y and Torquato S 2011 Phys. Rev. E 83051308

[329] Berthier L, Chaudhuri P, Coulais C, Dauchot O and Sollich P 2011 Phys. Rev. Lett. 106120601

[330] Kurita R and Weeks E R 2011 Phys. Rev. E 84 030401(R)

[331] Hodge I M 1995 Science 267 1945-7

[332] Kob W and Barrat J L 1997 Phys. Rev. Lett. 78 4581-4

[333] Kob W, Barrat J L, Sciortino F and Tartaglia P 2000 J. Phys.: Condens. Matter 12 6385-94

[334] Kob W and Barrat J L 2000 Eur. Phys. J. B 13 319-33

[335] Martinez V A, Bryant G and van Megen W 2008 Phys. Rev. Lett. 101135702

[336] Vollmayr-Lee K, Roman J A and Horbach J 2010 Phys. Rev. E 81061203

[337] Rottler J and Warren M 2008 Euro. Phys. J. Spec. Top. $16155-63$

[338] Boettcher S and Sibani P 2011 J. Phys.: Condens. Matter 23065103

[339] Masri D E, Pierno M, Berthier L and Cipelletti L 2005 J. Phys.: Condens. Matter 17 S3543-S3549

[340] Castillo H E, Chamon C, Cugliandolo L F and Kennett M P 2002 Phys. Rev. Lett. 88237201

[341] Cloitre M, Borrega R and Leibler L 2000 Phys. Rev. Lett. 85 4819-22

[342] Viasnoff V and Lequeux F 2002 Phys. Rev. Lett. 89065701

[343] Bonn D, Tanase S, Abou B, Tanaka H and Meunier J 2002 Phys. Rev. Lett. 89015701

[344] Derec C, Ducouret G, Ajdari A and Lequeux F 2003 Phys. Rev. Е 67061403

[345] Purnomo E H, van den Ende D, Mellema J and Mugele F 2006 Europhys. Lett. 76 74-80

[346] Cianci G C, Courtland R E and Weeks E R 2006 Solid State Commun. 139 599-604

[347] McKenna G B 2003 J. Phys.: Condens. Matter 15 S737-S763

[348] McKenna G B, Narita T and Lequeux F 2009 J. Rheol. 53 489-516

[349] Purnomo E H, van den Ende D, Mellema J and Mugele F 2007 Phys. Rev. E 76021404

[350] van den Ende D, Purnomo E H, Duits M H G, Richtering W and Mugele F 2010 Phys. Rev. E 81011404

[351] Purnomo E H, van den Ende D, Vanapalli S A and Mugele F 2008 Phys. Rev. Lett. 101238301

[352] Di X, Win K Z, McKenna G B, Narita T, Lequeux F, Pullela S R and Cheng Z 2011 Phys. Rev. Lett. 106095701

[353] Kovacs A J 1964 Fortschr. Hochpolym. Forsch. 3 394-507
[354] Zheng Y and McKenna G B 2003 Macromolecules 36 2387-96

[355] Tant M R and Wilkes G L 1981 Polym. Eng. Sci. 21 874-95

[356] Cianci G C, Courtland R E and Weeks E R 2006 AIP Conf. Proc. 832 21-5

[357] Buisson L, Bellon L and Ciliberto S 2003 J. Phys.: Condens. Matter 15 S1163-S1179

[358] Vollmayr-Lee K, Kob W, Binder K and Zippelius A 2002 J. Chem. Phys. 116 5158-66

[359] Vollmayr-Lee K and Zippelius A 2005 Phys. Rev. E 72041507

[360] Castillo H E and Parsaeian A 2007 Nature Phys. $326-8$

[361] Kob W, Sciortino F and Tartaglia P 2000 Europhys. Lett. 49 590-6

[362] Kawasaki T, Araki T and Tanaka H 2007 Phys. Rev. Lett. 99215701

[363] Royall C P, Williams S R, Ohtsuka T and Tanaka H 2008 Nature Mater. 7 556-61

[364] Cugliandolo L F, Kurchan J and Peliti L 1997 Phys. Rev. E 55 3898-914

[365] Segrè P N, Liu F, Umbanhowar P and Weitz D A 2001 Nature 409 594-7

[366] Ono I K, O'Hern C S, Durian D J, Langer S A, Liu A J and Nagel S R 2002 Phys. Rev. Lett. 89095703

[367] Song C, Wang P and Makse H A 2005 Proc. Natl Acad. Sci. USA 102 2299-304

[368] Wang P, Song C, Briscoe C and Makse H A 2008 Phys. Rev. E 77061309

[369] Kawasaki T and Tanaka H 2009 Phys. Rev. Lett. 102185701

[370] Grigera T S and Israeloff N E 1999 Phys. Rev. Lett. 83 5038-41

[371] Hérisson D and Ocio M 2002 Phys. Rev. Lett. 88257202

[372] Bellon L 2002 Physica D 168/169 325-35

[373] Bellon L, Ciliberto S and Laroche C 2001 Europhys. Lett. $53511-17$

[374] Wang P, Song C and Makse H A 2006 Nature Phys. 2 526-31

[375] Farouji S J, Mizuno D, Atakhorrami M, Mackintosh F C, Schmidt C F, Eiser E, Wegdam G H and Bonn D 2007 Phys. Rev. Lett. 98108302

[376] Assoud L, Ebert F, Keim P, Messina R, Maret G and Löwen H 2009 Phys. Rev. Lett. 102238301

[377] Koumakis N, Schofield A B and Petekidis G 2008 Soft Matter 4 2008-18

[378] Fielding S M, Cates M E and Sollich P 2009 Soft Matter $52378-82$

[379] Rogers R B and Lagerlöf K P D 2008 Appl. Opt. 47 284-95

[380] Chen D, Semwogerere D, Sato J, Breedveld V and Weeks E R 2010 Phys. Rev. E 81011403

[381] Petekidis G, Vlassopoulos D and Pusey P N 2003 Faraday Discuss. 123 287-302

[382] Petekidis G, Vlassopoulos D and Pusey P N 2004 J. Phys. Condens. Matter 16 S3955-S3963

[383] Eisenmann C, Kim C, Mattsson J and Weitz D A 2010 Phys. Rev. Lett. 104035502

[384] Maloney C E and Robbins M O 2008 J. Phys.: Condens. Matter 20244128

[385] Schall P, Weitz D A and Spaepen F 2007 Science 318 1895-9

[386] Petekidis G, Moussaid A and Pusey P N 2002 Phys. Rev. E 66051402

[387] Besseling R, Weeks E R, Schofield A B and Poon W C K 2007 Phys. Rev. Lett. 99028301

[388] Zausch J, Horbach J, Laurati M, Egelhaaf S U, Brader J M, Voigtmann T and Fuchs M 2008 J. Phys.: Condens. Matter 20404210

[389] Chikkadi V, Wegdam G, Bonn D, Nienhuis B and Schall P 2011 Phys. Rev. Lett. 107198303

[390] Shiba H and Onuki A 2010 Phys. Rev. E 81051501

[391] Furukawa A, Kim K, Saito S and Tanaka H 2009 Phys. Rev. Lett. 102016001 
[392] Falk M L and Langer J S 1998 Phys. Rev. E 57 7192-205

[393] Manneville S 2008 Rheol. Acta 47 301-18

[394] Ovarlez G, Rodts S, Chateau X and Coussot P 2009 Rheol. Acta 48 831-44

[395] Schall P and van Hecke M 2010 Annu. Rev. Fluid Mech. 42 67-88

[396] Dhont J K G 1999 Phys. Rev. E 60 4534-44

[397] Dhont J K G, Lettinga M P, Dogic Z, Lenstra T A J, Wang H, Rathgeber S, Carletto P, Willner L, Frielinghaus $\mathrm{H}$ and Lindner P 2003 Faraday Discuss. 123 157-72

[398] Dhont J and Briels W 2008 Rheol. Acta 47 257-81

[399] Moller P C F, Rodts S, Michels M A J and Bonn D 2008 Phys. Rev. E 77041507

[400] Besseling R, Isa L, Ballesta P, Petekidis G, Cates M E and Poon W C K 2010 Phys. Rev. Lett. 105268301

[401] Ballesta P, Besseling R, Isa L, Petekidis G and Poon W C K 2008 Phys. Rev. Lett. 101258301

[402] Divoux T, Tamarii D, Barentin C and Manneville S 2010 Phys. Rev. Lett. 104208301

[403] Hébraud P, Lequeux F, Munch J P and Pine D J 1997 Phys. Rev. Lett. 78 4657-60

[404] Le Grand A and Petekidis G 2008 Rheol. Acta 47 579-90

[405] Rogers R B and Ackerson B J 2010 Phil. Mag. 91 682-729

[406] Smith P A, Petekidis G, Egelhaaf S U and Poon W C K 2007 Phys. Rev. E 76041402

[407] Pham K N, Petekidis G, Vlassopoulos D, Egelhaaf S U, Poon W C K and Pusey P N 2008 J. Rheol. 52 649-76

[408] Zaccarelli E and Poon W C K 2009 Proc. Natl Acad. Sci. USA 106 15203-8

[409] Sollich P, Lequeux F, Hébraud P and Cates M E 1997 Phys. Rev. Lett. 78 2020-3

[410] Brader J M, Voigtmann T, Cates M E and Fuchs M 2007 Phys. Rev. Lett. 98058301

[411] Goyon J, Colin A, Ovarlez G, Ajdari A and Bocquet L 2008 Nature 454 84-7

[412] Bocquet L, Colin A and Ajdari A 2009 Phys. Rev. Lett. 103036001

[413] Katgert G, Tighe B P, Möbius M E and van Hecke M 2010 Europhys. Lett. 9054002

[414] Nichol K, Zanin A, Bastien R, Wandersman E and van Hecke M 2010 Phys. Rev. Lett. 104078302

[415] Chen K, Ellenbroek W G, Zhang Z, Chen D T N, Yunker P J, Henkes S, Brito C, Dauchot O, van Saarloos W, Liu A J and Yodh A G 2010 Phys. Rev. Lett. 105025501

[416] Dingenouts N, Norhausen C and Ballauff M 1998 Macromolecules 31 8912-8917

[417] Crassous J J, Wittemann A, Siebenbürger M, Schrinner M, Drechsler M and Ballauff M 2008 Colloid Polym. Sci. 286 805-12

[418] Jones C D and Lyon L A 2000 Macromolecules 33 8301-6

[419] Asakura S and Oosawa F 1954 J. Chem. Phys. 22 1255-6

[420] Fabbian L, Götze W, Sciortino F, Tartaglia P and Thiery F 1999 Phys. Rev. E 59 R1347-R1350

[421] Fabbian L, Götze W, Sciortino F, Tartaglia P and Thiery F 1999 Phys. Rev. E 602430

[422] Bergenholtz J and Fuchs M 1999 Phys. Rev. E 59 5706-15

[423] Dawson K, Foffi G, Fuchs M, Götze W, Sciortino F, Sperl M, Tartaglia P, Voigtmann Th and Zaccarelli E 2000 Phys. Rev. E 63011401

[424] Puertas A M, Fuchs M and Cates M E 2002 Phys. Rev. Lett. 88098301

[425] Pham K N, Puertas A M, Bergenholtz J, Egelhaaf S U, Moussaid A, Pusey P N, Schofield A B, Cates M E, Fuchs $M$ and Poon W C K 2002 Science 296 104-6

[426] Pham K N, Egelhaaf S U, Pusey P N and Poon W C K 2004 Phys. Rev. E 69011503

[427] Lu P J, Zaccarelli E, Ciulla F, Schofield A B, Sciortino F and Weitz D A 2008 Nature $\mathbf{4 5 3}$ 499-503
[428] Lekkerkerker H N W, Poon W C K, Pusey P N, Stroobants A and Warren P B 1992 Europhys. Lett. 20 559-64

[429] Ilett S M, Orrock A, Poon W C K and Pusey P N 1995 Phys. Rev. E 51 1344-52

[430] Poon W C K 2002 J. Phys.: Condens. Matter 14 R859-R880

[431] Dawson K A 2002 Curr. Opin. Colloid Interface Sci. $7218-27$

[432] Mourchid A, Delville A, Lambard J, LeColier E and Levitz P 1995 Langmuir 11 1942-50

[433] Mourchid A, Lécolier E, Van Damme H and Levitz P 1998 Langmuir 14 4718-23

[434] Bonn D, Tanaka H, Wegdam G, Kellay H and Meunier J 1999 Europhys. Lett. 45 52-7

[435] Abou B, Bonn D and Meunier J 2001 Phys. Rev. E 64021510

[436] Joshi Y M 2007 J. Chem. Phys. 127081102

[437] Bellour M, Knaebel A, Harden J L, Lequeux F and Munch J P 2003 Phys. Rev. E 67031405

[438] Bandyopadhyay R, Liang D, Yardimci H, Sessoms D A, Borthwick M A, Mochrie S G J, Harden J L and Leheny R L 2004 Phys. Rev. Lett. 93228302

[439] Bibette J, Calderon L F and Poulin P 1999 Rep. Prog. Phys. 62 969-1033

[440] Mason T G, Wilking J N, Meleson K, Chang C B and Graves S M 2006 J. Phys.: Condens. Matter 18 R635-R666

[441] Mason T G, Bibette J and Weitz D A 1995 Phys. Rev. Lett. 75 2051-4

[442] Mason T G, Lacasse M D, Grest G S, Levine D, Bibette J and Weitz D A 1997 Phys. Rev. E 56 3150-66

[443] Brujić J, Edwards S F, Grinev D V, Hopkinson I, Brujic D and Makse H A 2003 Faraday Discuss. 123 207-20

[444] Brujić J, Edwards S F, Hopkinson I and Makse H 2003 Physica A 327 201-12

[445] Zhou J, Long S, Wang Q and Dinsmore A D 2006 Science 312 1631-3

[446] Brujić J, Song C, Wang P, Briscoe C, Marty G and Makse H A 2007 Phys. Rev. Lett. 98248001

[447] Clusel M, Corwin E I, Siemens A O N and Brujić J 2009 Nature 460 611-5

[448] O'Hern C S, Langer S A, Liu A J and Nagel S R 2001 Phys. Rev. Lett. 86 111-4

[449] Katgert G and van Hecke M 2010 Europhys. Lett. 9234002

[450] Durian D J 1995 Phys. Rev. Lett. 75 4780-3

[451] Saint-Jalmes A and Durian D J 1999 J. Rheol. 43 1411-22

[452] Langer S A and Liu A J 2000 Europhys. Lett. 49 68-74

[453] Debrégeas G, Tabuteau H and di Meglio J M 2001 Phys. Rev. Lett. 87178305

[454] Lauridsen J, Twardos M and Dennin M 2002 Phys. Rev. Lett. 89098303

[455] Kabla A and Debrégeas G 2003 Phys. Rev. Lett. 90258303

[456] Dennin M 2004 Phys. Rev. E 70041406

[457] Lundberg M, Krishan K, Xu N, O'Hern C S and Dennin M 2008 Phys. Rev. E 77041505

[458] Edwards S F and Anderson P W 1975 J. Phys. F: Met. Phys. 5 965-74

[459] Chamon C, Cugliandolo L F, Fabricius G, Iguain J L and Weeks E R 2008 Proc. Natl Acad. Sci. USA 105 15263-8

[460] Manoharan V N, Elsesser M T and Pine D J 2003 Science $301483-7$

[461] Yi G R, Manoharan V N, Michel E, Elsesser M T, Yang S M and Pine D J 2004 Adv. Mater. 16 1204-8

[462] Mohraz A and Solomon M J 2005 Langmuir 21 5298-306

[463] Champion J A, Katare Y K and Mitragotri S 2007 Proc. Natl Acad. Sci. USA 104 11901-4

[464] Gerbode S J, Lee S H, Liddell C M and Cohen I 2008 Phys. Rev. Lett. 101058302

[465] Elsesser M T, Hollingsworth A D, Edmond K V and Pine D J 2011 Langmuir 27 917-27 
[466] Zheng Z, Wang F and Han Y 2011 Phys. Rev. Lett. 107065702

[467] Schilling R and Scheidsteger T 1997 Phys. Rev. E $562932-49$

[468] Letz M, Schilling R and Latz A 2000 Phys. Rev. E 62 5173-8

[469] Yatsenko G and Schweizer K S 2007 Phys. Rev. E 76041506

[470] De Michele C, Schilling R and Sciortino F 2007 Phys. Rev. Lett. 98265702

[471] Pfleiderer P, Milinkovic K and Schilling T 2008 Europhys. Lett. 8416003
[472] Weeks E R 2011 Physics 461

[473] Bianchi E, Largo J, Tartaglia P, Zaccarelli E and Sciortino F 2006 Phys. Rev. Lett. 97168301

[474] Zaccarelli E 2007 J. Phys.: Condens. Matter 19323101

[475] Bianchi E, Blaak R and Likos C N 2011 Phys. Chem. Chem. Phys. 13 6397-410

[476] Anthony S M, Hong L, Kim M and Granick S 2006 Langmuir 22 9812-5

[477] Glotzer S C and Solomon M J 2007 Nature Mater. 6 557-62

[478] Pusey P N 2008 J. Phys.: Condens. Matter 20494202 